\title{
Recovery of a space-dependent vector source in anisotropic thermoelastic systems
}

\author{
Karel Van Bockstal ${ }^{\mathrm{a}, *}$, Liviu Marin ${ }^{\mathrm{b}, \mathrm{c}}$ \\ ${ }^{a}$ Research Group NaM2, Department of Mathematical Analysis, Ghent University, Krijgslaan 281, 9000 Ghent, Belgium \\ ${ }^{b}$ Department of Mathematics, Faculty of Mathematics and Computer Science, University of Bucharest, 14 Academiei, 010014 \\ Bucharest, Romania \\ ${ }^{c}$ Institute of Solid Mechanics, Romanian Academy, 15 Constantin Mille, 010141 Bucharest, Romania
}

\begin{abstract}
We investigate the theoretical and numerical determination of a space-dependent vector source (load) in an anisotropic thermoelastic system of type-III form the knowledge of an additional final time measurement. The uniqueness of a solution to this inverse source problem is proved for various assumptions made on the convolution kernel. A convergent and stable iterative algorithm is proposed for the recovery of the unknown vector source in the linear case and, at the same time, a stopping criterion is also given. Three numerical experiments are considered to validate the properties of the proposed iterative procedure and the regularizing/stabilizing character of the corresponding stopping criterion. The numerical experiments carried out showed that it exists a certain limitation of the method with respect to the recovery of nonsymmetric sources.
\end{abstract}

Keywords: anisotropic thermoelasticity, inverse problems, iterative regularization, discrepancy principle, finite element method

2010 MSC: 35Q61, 35K61, 35R09, 65M20, 82D55

\section{Introduction}

In numerous practical applications related to nuclear power plants, engines and electronic devices, structures of aircraft and propulsion systems, gas and steam turbines, or in chemical reactors, the effect of thermo-mechanical loads acting on the solid body of interest must be studied and analyzed. In such situations, thermal stresses may arise in a heated body because of a non-uniform temperature distribution, external constraints, or a combination of these conditions. Also, the cooling and heating of a solid may be associated with a change of volume and, consequently, the temperature distribution in the body is influenced by the diagonal components of the strain tensor [1]. Green and Naghdi [2] described the heat flow in solid bodies by employing a general entropy balance. According to these authors, the characterization of material response for such thermal phenomena is referred to as type-I, type-II and type-III thermoelasticity. After linearization, the type-I thermoelasticity actually coincides with the classical heat conduction theory (Fourier's law). This theory has the shortcoming that a thermal disturbance at one point of the body is instantly felt

\footnotetext{
${ }^{*}$ Corresponding author

Email addresses: Karel.VanBockstal@UGent.be (Karel Van Bockstal), marin.liviulgmail.com, liviu.marinefmi.unibuc.ro(Liviu Marin)

URL: http://cage. UGent.be/ kvb/ (Karel Van Bockstal), +32 92644872 (Karel Van Bockstal), https://sites.google.com/site/marinliviu/home/(Liviu Marin)
} 
everywhere (i.e. infinite speed of propagation). This is physically not acceptable for materials with memory and is overcome by taking into account memory effects in the models for type-II and type-III thermoelasticity, with the mention that the heat conduction is independent of the present values of the temperature gradient in type-II thermoelasticity as compared to type-III thermoelasticity.

Direct problems in thermoelasticity have been thoroughly investigated in the literature and are, in general, characterized by the knowledge of (i) the governing partial differential equations; (ii) the geometry of the domain of interest; (iii) the material properties; (iv) the boundary and/or initial conditions; and (v) the magnitudes and locations of any possible sources of stress, deformation, or heat inside or on the boundary of the domain. If at least one of the aforementioned conditions is entirely or partially unknown, then one deals with a so-called inverse problem associated with thermoelastic materials. It is well-known that most of these inverse problems are ill-posed in the sense of Hadamard [3], meaning that small errors in the boundary and/or initial data may significantly amplify the errors in the solution. Consequently, inverse problems in solid mechanics are more difficult to solve than direct problems.

The inverse problems in thermoelasticity addressed so far in the literature are mainly related to boundary data reconstruction, shape optimization problems, detection of flaws (e.g. inclusions, cavities and cracks), identification of material parameters and inverse source or load reconstruction problems, see e.g. [4-16]. Dennis et al. [4, 5] proposed a regularized finite element method formulation for the determination of unknown thermal and elasticity boundary conditions in 2D and 3D steady problems, respectively. The reconstruction of the missing thermal and mechanical fields on an inaccessible part of the boundary for linear isotropic thermoelastic materials from over-prescribed noisy data on the remaining accessible boundary was been studied in two- and three-dimensions by Tanaka et al. [17], Marin and Karageorghis [6], Karageorghis et al. [7] and Marin et al. [8]. Yu [9] determined the thermomechanical parameters for the quasistatic thermoelasticity system with over-specified data by employing a minimization functional, whilst Dennis et al. [10] investigated the inverse detection of thermoelasticity coefficients in 2D plates. Nedin et. al. [11] used the operator equations in inverted transforms and an iterative procedure for reconstructing the thermoelastic coefficients of an inhomogeneous rod.

Although a vast majority of inverse source (load reconstruction) problems in solid mechanics have been investigated for either the thermal or the mechanical process, it should be mentioned that several authors have studied such inverse problems for thermoelastic processes in recent years. Bellassoued and Yamamoto [12] investigated an inverse heat source problem for type-I thermoelasticity without accounting for memory effects. On using a Carleman estimate, these authors prove a Hölder stability for the inverse source problem, which implies the uniqueness of the inverse source problem. An inverse source problem for type-II thermoelasticity that consists of the determination of a space-dependent vector source (load), was investigated by $\mathrm{Wu}$ and Liu [13]. Again, based on a Carleman estimate, a Hölder stability for the inverse source problem was obtained from a displacement measurement. However, we note that no numerical schemes were provided to recover the unknown sources in [12, 13]. Van Bockstal and Slodička [14] proposed a stable iterative algorithm to recover a space-dependent vector load (source) for all types of thermoelasticity from the knowledge of an additional measurement at the final time. The uniqueness and stability of a spatially varying thermal kernel function in a thermoelastic system of type-III was investigated by Wu et al. [15] by using a Carleman estimate. Recently, Van Bockstal and Slodička [16] addressed the theoretical and numerical determination of a time-dependent heat source in a thermoelastic system of type-III from an additional global measurement.

Encouraged by the promising results obtained by Van Bockstal and Slodička [14] for the reconstruction of a space-dependent vector load (source) in an isotropic thermoelastic system of types I-III from the knowledge of an additional measurement at the final time, in this study we investigate the same inverse 
source problem but in the framework of an anisotropic thermoelastic system of type-III. The paper is organized as follows. In Section 2 we present the inverse source problem under investigation together with the notations used herein. For various assumptions on the convolution kernel, the uniqueness of a solution to the inverse problem addressed herein is established Section 3. A convergent and stable iterative algorithm for the recovery of the unknown vector source in the linear case and a regularizing stopping criterion are proposed in Section 4. Three numerical examples are considered and thoroughly investigated in Section 5. Finally, some concluding remarks and ideas for future work are presented in Section 6

\section{Mathematical formulation of the problem}

Consider an anisotropic homogeneous thermoelastic body occupying an open and bounded domain $\Omega \subset$ $\mathbb{R}^{d}$ with a Lipschitz continuous boundary $\Gamma$, where $d \in\{1,2,3\}$ is the dimension of the space where the problem is posed. Let $Q_{T}=\Omega \times(0, T)$ and $\Sigma_{T}=\Gamma \times(0, T)$, where $T>0$ is a given final time. The convolution product of a kernel $K$ and a function $\theta$ is denoted by

$$
(K * \theta)(\mathbf{x}, t):=\int_{0}^{t} K(t-s) \theta(\mathbf{x}, s) \mathrm{d} s, \quad(\mathbf{x}, t) \in Q_{T} .
$$

The divergence of a continuously differentiable second-order tensor field $\mathbb{A}$ is, by definition, the first-order tensor field given by

$$
\operatorname{div} \mathbb{A}=\left[\partial_{j} A_{1 j}, \partial_{j} A_{2 j}, \ldots, \partial_{j} A_{d j}\right]^{\top} \in \mathbb{R}^{d},
$$

where $\partial_{j}:=\partial / \partial x_{j}, 1 \leq j \leq d$, and Einstein's notation, i.e. summation of the repeated indices, is used in the following. Moreover, the scalar product of two second-order tensor fields $\mathbb{A}$ and $\mathbb{B}$ is denoted by

$$
\mathbb{A}: \mathbb{B}=A_{i j} B_{i j} \in \mathbb{R} .
$$

In the sequel, the following anisotropic thermoelastic system (of type-III), describing both the elastic and the thermal behaviours in $\Omega$, is considered

$$
\begin{cases}\varrho(\mathbf{x}) \partial_{t t} \mathbf{u}(\mathbf{x}, t)+\mathbf{g}\left(\partial_{t} \mathbf{u}(\mathbf{x}, t)\right)+\mathcal{L}^{\mathrm{e}} \mathbf{u}(\mathbf{x}, t)+\operatorname{div}(\mathbb{B}(\mathbf{x}) \theta(\mathbf{x}, t))=\mathbf{p}(\mathbf{x})+\mathbf{r}(\mathbf{x}, t), & (\mathbf{x}, t) \in Q_{T}, \\ \varrho(\mathbf{x}) C_{s}(\mathbf{x}) \partial_{t} \theta(\mathbf{x}, t)-\nabla \cdot(\mathbb{K}(\mathbf{x}) \nabla \theta(\mathbf{x}, t))-(K * \Delta \theta)(\mathbf{x}, t)+T_{0} \mathbb{B}(\mathbf{x}): \nabla \partial_{t} \mathbf{u}(\mathbf{x}, t)=h(\mathbf{x}, t), & (\mathbf{x}, t) \in Q_{T}, \\ \mathbf{u}(\mathbf{x}, t)=\mathbf{0}, & (\mathbf{x}, t) \in \Sigma_{T}, \\ \theta(\mathbf{x}, t)=0, & (\mathbf{x}, t) \in \Sigma_{T},\end{cases}
$$

together with the initial conditions

$$
\mathbf{u}(\mathbf{x}, 0)=\overline{\mathbf{u}}_{0}(\mathbf{x}), \quad \partial_{t} \mathbf{u}(\mathbf{x}, 0)=\overline{\mathbf{u}}_{1}(\mathbf{x}), \quad \theta(\mathbf{x}, 0)=\bar{\theta}_{0}(\mathbf{x}), \quad \mathbf{x} \in \Omega .
$$

Here, the vector source $\mathbf{p}$ (load vector) is assumed to be unknown and depend solely on the space variable, whilst both the vector source $\mathbf{r}$ and the heat source $h$ are assumed to be known. Furthermore, $\mathbf{u}=\left(u_{1}, \ldots, u_{d}\right)^{\top}$ and $\theta$ denote the displacement and the temperature difference from the reference value $T_{0}>0$ (in Kelvin), respectively, of the thermoelastic material at point $\mathrm{x}$ and time $t$, with the mention that the reference temperature corresponds to the undeformed and unstressed state of the material.

The goal of this paper is to determine the spatial vector function $\mathbf{p}(\mathbf{x})$ from the knowledge of an additional measurement, more specifically, the condition of final over-determination:

$$
\mathbf{u}_{T}(\mathbf{x}):=\mathbf{u}(\mathbf{x}, T)=\boldsymbol{\xi}_{T}(\mathbf{x}), \quad \mathbf{x} \in \Omega .
$$

In the sequel, the following assumptions are made: 
(a) The mass density $\rho$ and the specific heat $C_{s}$ of the material are bounded, i.e.

$$
\exists \varrho_{1} \geq \varrho_{0}>0 \quad \text { s.t. } \varrho_{0} \leqslant \varrho(\mathbf{x}) \leqslant \varrho_{1}, \quad \text { a.e. in } \Omega
$$

and

$$
\exists c_{1} \geq c_{0}>0 \quad \text { s.t. } \quad c_{0} \leqslant C_{s}(\mathbf{x}) \leqslant c_{1}, \quad \text { a.e. in } \Omega .
$$

(b) Small displacements are assumed, i.e. the strain tensor $\varepsilon=\left(\varepsilon_{i j}\right)_{1 \leq i, j \leq d}$ is defined by the kinematic relations

$$
\varepsilon_{i j}(\mathbf{u}(\mathbf{x}, t))=\frac{1}{2}\left[\partial_{j} u_{i}(\mathbf{x}, t)+\partial_{i} u_{j}(\mathbf{x}, t)\right], \quad 1 \leq i, j \leq d .
$$

(c) The stress tensor $\boldsymbol{\sigma}=\left(\sigma_{i j}\right)_{1 \leq i, j \leq d}$ is related to the strain tensor $\boldsymbol{\varepsilon}=\left(\varepsilon_{i j}\right)_{1 \leq i, j \leq d}$ by a Hooke's-type constitutive law, i.e.

$$
\sigma_{i j}(\mathbf{x}, t)=C_{i j k l}(\mathbf{x}) \varepsilon_{k l}(\mathbf{x}, t), \quad 1 \leqslant i, j, k, l \leqslant d,
$$

where the components of the fourth-order elasticity tensor $C_{i j k l}, 1 \leq i, j, k, l \leq d$, satisfy the following conditions [18]:

(i) $C_{i j k l} \in \mathrm{L}^{\infty}(\Omega)$, i.e. $\exists M>0$ s.t. $\max _{1 \leqslant i, j, k, l \leqslant d}\left\|C_{i j k l}\right\|_{\mathrm{L}^{\infty}(\Omega)} \leqslant M$;

(ii) $C_{i j k l}(\mathbf{x})=C_{k l i j}(\mathbf{x})=C_{j i k l}(\mathbf{x})$ a.e in $\Omega, 1 \leqslant i, j, k, l \leqslant d$;

(iii) $\exists m>0$ s.t. $C_{i j k l}(\mathbf{x}) \boldsymbol{\tau}_{i j} \boldsymbol{\tau}_{k l} \geqslant m \boldsymbol{\tau}_{i j} \boldsymbol{\tau}_{i j}$, a.e. in $\Omega, \forall \boldsymbol{\tau} \in \mathbb{R}^{d \times d}$ with $\boldsymbol{\tau}_{i j}=\boldsymbol{\tau}_{j i}$.

Define

$$
a: \mathbf{H}_{0}^{1}(\Omega) \times \mathbf{H}_{0}^{1}(\Omega) \rightarrow \mathbb{R}, \quad a(\mathbf{u}, \mathbf{v})=\int_{\Omega} C_{i j k l}(\mathbf{x}) \partial_{l} u_{k}(\mathbf{x}) \partial_{j} v_{i}(\mathbf{x}) \mathrm{d} \mathbf{x} .
$$

Then $a$ is a symmetric, continuous and $\mathbf{H}_{0}^{1}(\Omega)$-elliptic bilinear form, i.e.

$$
\begin{array}{ll}
a(\mathbf{u}, \mathbf{v})=a(\mathbf{v}, \mathbf{u}), & \forall \mathbf{u}, \mathbf{v} \in \mathbf{H}_{0}^{1}(\Omega), \\
|a(\mathbf{u}, \mathbf{v})| \leqslant M\|\mathbf{u}\|_{\mathbf{H}_{0}^{1}(\Omega)}\|\mathbf{v}\|_{\mathbf{H}_{0}^{1}(\Omega)}, & \forall \mathbf{u}, \mathbf{v} \in \mathbf{H}_{0}^{1}(\Omega), \\
a(\mathbf{u}, \mathbf{u}) \geqslant m\|\mathbf{u}\|_{\mathbf{H}_{0}^{1}(\Omega)}^{2}, & \forall \mathbf{u} \in \mathbf{H}_{0}^{1}(\Omega) .
\end{array}
$$

If $\langle\cdot, \cdot\rangle$ denotes the duality pairing on $\mathbf{H}_{0}^{1}(\Omega)^{*} \times \mathbf{H}_{0}^{1}(\Omega)$, then the bilinear form $a$ can be used to define the elasticity operator $\mathcal{L}^{\mathrm{e}}: \mathbf{H}_{0}^{1}(\Omega) \rightarrow \mathbf{H}_{0}^{1}(\Omega)^{*}$ by

$$
\left\langle\mathcal{L}^{\mathrm{e}} \mathbf{u}, \mathbf{v}\right\rangle=a(\mathbf{u}, \mathbf{v}), \quad \forall \mathbf{u}, \mathbf{v} \in \mathbf{H}_{0}^{1}(\Omega) .
$$

Formally, the operator $\mathcal{L}^{\mathrm{e}}$ is defined by

$$
\left(\mathcal{L}^{\mathrm{e}} \mathbf{u}\right)_{i}=-\left(C_{i j k l} u_{k, l}\right)_{, j}=-(\operatorname{div} \boldsymbol{\sigma})_{i}, \quad 1 \leq i \leq d .
$$

In view of the properties of $a$, the operator $\mathcal{L}^{\mathrm{e}}$ is linear, continuous and $\mathbf{H}_{0}^{1}(\Omega)$-elliptic. Moreover, the following important relationship holds

$$
\partial_{t}\left(\mathcal{L}^{\mathrm{e}} \mathbf{u}, \mathbf{u}\right)=\partial_{t} a(\mathbf{u}, \mathbf{u})=a\left(\partial_{t} \mathbf{u}, \mathbf{u}\right)+a\left(\mathbf{u}, \partial_{t} \mathbf{u}\right)=2 a\left(\mathbf{u}, \partial_{t} \mathbf{u}\right)=2\left(\mathcal{L}^{\mathrm{e}} \mathbf{u}, \partial_{t} \mathbf{u}\right),
$$

where $(\cdot, \cdot)$ denotes the $\mathrm{L}^{2}(\Omega)$ inner product; 
(d) The thermal conductivity tensor $\mathbb{K}(\mathbf{x})=\left(K_{i j}(\mathbf{x})\right)_{1 \leq i, j \leq d}$ is symmetric and positive definite, i.e.

$$
\exists k_{0}>0 \quad \text { s.t. } \quad K_{i j}(\mathbf{x}) \boldsymbol{\xi}_{i} \boldsymbol{\xi}_{j} \geqslant k_{0}|\boldsymbol{\xi}|^{2}, \quad \text { a.e. in } \Omega, \quad \forall \boldsymbol{\xi} \in \mathbb{R}^{d} .
$$

Moreover, $\mathbb{K} \in \mathrm{L}^{\infty}(\Omega)^{d \times d}$, i.e.

$$
\exists k_{1}>0 \quad \text { s.t. } \max _{1 \leqslant i, j \leqslant d}\left\|K_{i j}\right\|_{\mathrm{L}^{\infty}(\Omega)} \leqslant k_{1}
$$

(e) The thermoelasticity tensor $\mathbb{B}(\mathbf{x})=\left(b_{i j}(\mathbf{x})\right)_{1 \leq i, j \leq d}$ belongs to $\mathrm{L}^{\infty}(\Omega)^{d \times d}$, i.e.

$$
\exists B_{1}>0 \quad \text { s.t. } \max _{1 \leqslant i, j \leqslant d}\left\|b_{i j}\right\|_{\mathrm{L}^{\infty}(\Omega)} \leqslant B_{1} .
$$

The thermoelasticity tensor is symmetric, i.e. $\mathbb{B}(\mathbf{x})=\mathbb{B}^{\top}(\mathbf{x})$. Moreover, on assuming

$$
\operatorname{div} \mathbb{B}=\mathbf{0},
$$

then

$$
\operatorname{div}(\mathbb{B} \theta)=\theta \operatorname{div} \mathbb{B}+\mathbb{B} \nabla \theta=\mathbb{B} \nabla \theta,
$$

and

$$
\operatorname{div}\left(\mathbb{B} \partial_{t} \mathbf{u}\right)=\mathbb{B}: \nabla \partial_{t} \mathbf{u}+\partial_{t} \mathbf{u} \cdot \operatorname{div} \mathbb{B}=\mathbb{B}: \nabla \partial_{t} \mathbf{u} .
$$

(f) The continuous nonlinear damping term $\mathrm{g}: \mathbb{R}^{d} \mapsto \mathbb{R}^{d}$ is strictly monotonically increasing, i.e.

$$
\left(\mathbf{g}\left(\mathbf{s}_{1}\right)-\mathbf{g}\left(\mathbf{s}_{2}\right)\right) \cdot\left(\mathbf{s}_{1}-\mathbf{s}_{2}\right) \geqslant 0, \quad \forall \mathbf{s}_{1}, \mathbf{s}_{2} \in \mathbb{R}^{d}
$$

and

$$
\left(\mathbf{g}\left(\mathbf{s}_{1}\right)-\mathbf{g}\left(\mathbf{s}_{2}\right)\right) \cdot\left(\mathbf{s}_{1}-\mathbf{s}_{2}\right)=0 \Rightarrow \mathbf{s}_{1}=\mathbf{s}_{2} .
$$

For instance, $\mathbf{g}(\mathbf{x})=a|\mathbf{x}|^{\alpha} \mathbf{x}$, with $a>0$, is strictly monotone for $\alpha \geqslant 0$.

(g) Various assumptions on the convolution kernel $K$ will be considered later.

Remark 2.1 (Other notations). We denote by $(\cdot, \cdot)$ the standard inner product in $\mathrm{L}^{2}(\Omega)$ and by $\|\cdot\|$ its induced norm. Let $X$ be an abstract Banach space endowed with the norm $\|\cdot\|_{X}$. Assume that $k \in \mathbb{N} \cup\{0\}$. The set of $k$-times continuously differentiable functions $w:[0, T] \rightarrow X$ equipped with the usual norm

$$
\sum_{j=0}^{k} \max _{t \in[0, T]}\left\|w^{(j)}(t)\right\|_{X}
$$

is denoted by $\mathrm{C}^{k}([0, T], X)$. The space $\mathrm{L}^{p}((0, T), X)$ is endowed with the norm $\left(\int_{0}^{T}\|\cdot\|_{X}^{p}\right)^{\frac{1}{p}}$ with $p>1$. The symbol $X^{*}$ stands for the dual space of $X$. Finally, $C, \varepsilon$ and $C_{\varepsilon}$ denote generic positive constants depending only on a priori known quantities, where $\varepsilon$ is small and $C_{\varepsilon}=C\left(\varepsilon^{-1}\right)$ is large. 


\section{Uniqueness results}

Using Green's formulae (5) and (6), the following coupled variational formulation for (1) is obtained:

Given $\mathbf{r}:(0, T] \rightarrow \mathbf{L}^{2}(\Omega)$ in $\mathrm{L}^{2}\left((0, T), \mathbf{L}^{2}(\Omega)\right), h:(0, T] \rightarrow \mathrm{L}^{2}(\Omega)$ in $\mathrm{L}^{2}\left((0, T), \mathrm{L}^{2}(\Omega)\right), \overline{\mathbf{u}}_{0}(\mathbf{x}) \in$ $\mathbf{H}^{1}(\Omega), \overline{\mathbf{u}}_{1}(\mathbf{x}) \in \mathbf{L}^{2}(\Omega), \bar{\theta}_{0} \in \mathrm{H}^{1}(\Omega)$ and $\boldsymbol{\xi}_{T} \in \mathbf{L}^{2}(\Omega)$, find $(\mathbf{u}(t), \theta(t), \mathbf{p}) \in \mathbf{H}_{0}^{1}(\Omega) \times \mathrm{H}_{0}^{1}(\Omega) \times \mathbf{L}^{2}(\Omega)$, with $\partial_{t} \mathbf{u}(t) \in \mathbf{L}^{2}(\Omega), \partial_{t t} \mathbf{u}(t) \in \mathbf{H}_{0}^{1}(\Omega)^{*}$ and $\partial_{t} \theta(t) \in \mathrm{H}_{0}^{1}(\Omega)^{*}$, such that

$$
\begin{aligned}
& \left(\varrho \partial_{t t} \mathbf{u}(t), \boldsymbol{\varphi}\right)+\left(\mathbf{g}\left(\partial_{t} \mathbf{u}(t)\right), \boldsymbol{\varphi}\right)+\left(\mathcal{L}^{\mathrm{e}} \mathbf{u}(t), \boldsymbol{\varphi}\right)+(\mathbb{B} \nabla \theta(t), \boldsymbol{\varphi})=(\mathbf{p}+\mathbf{r}(t), \boldsymbol{\varphi}) \\
& \left(\varrho C_{s} \partial_{t} \theta(t), \psi\right)+(\mathbb{K} \nabla \theta(t), \nabla \psi)+((K * \nabla \theta)(t), \nabla \psi)-T_{0}\left(\mathbb{B} \partial_{t} \mathbf{u}(t), \nabla \psi\right)=(h(t), \psi)
\end{aligned}
$$

for all $\varphi \in \mathbf{H}_{0}^{1}(\Omega)$ and $\psi \in \mathrm{H}_{0}^{1}(\Omega)$ and a.a. $t \in[0, T]$.

The space-dependent measurement (3) ensures that the inverse problem has a unique solution. This is stated in the following three theorems, wherein various assumptions on the convolution kernel $K$ are made.

Theorem 3.1 (Uniqueness for a singular and positive definite convolution kernel). Suppose that the twice differentiable function $K:(0, T] \rightarrow \mathbb{R}$ satisfies

$$
K^{\prime}(t) \not \equiv 0 \quad \text { and } \quad(-1)^{j} K^{(j)}(t) \geqslant 0, \quad t>0, \quad j=0,1,2,
$$

i.e. $K$ is strongly positive definite [19] Corollary 7.2.1], which is equivalent with the existence of a positive constant $C_{0}$ independent of $T$ such that [19. Lemma 7.2.2]-[20. 21]

$$
\int_{0}^{T} \phi(t)(k * \phi)(t) \mathrm{d} t \geqslant C_{0} \int_{0}^{T}(k * \phi)^{2}(t) \mathrm{d} t, \quad \forall T>0, \forall \phi \in \mathrm{L}_{\mathrm{loc}}^{1}(0, T) .
$$

Then, there exists at most one $\mathbf{p} \in \mathbf{L}^{2}(\Omega)$ such that problem (1) and condition (3) hold.

Proof. A classical variational approach is used to establish the uniqueness of solution. The crucial points in the proof are the integration by parts formulae

$$
\int_{0}^{T}\left(\mathcal{L}^{\mathrm{e}} \mathbf{u}(t), \partial_{t} \mathbf{u}(t)\right) \mathrm{d} t \stackrel{4}{=} \frac{1}{2}\left(\mathcal{L}^{\mathrm{e}} \mathbf{u}(T), \mathbf{u}(T)\right)-\frac{1}{2}\left(\mathcal{L}^{\mathrm{e}} \mathbf{u}(0), \mathbf{u}(0)\right)
$$

and

$$
\int_{0}^{T} \mathbf{p}(\mathbf{x}) \cdot \partial_{t} \mathbf{u}(\mathbf{x}, t) \mathrm{d} t=\mathbf{p}(\mathbf{x}) \cdot \mathbf{u}(\mathbf{x}, T)-\mathbf{p}(\mathbf{x}) \cdot \mathbf{u}(\mathbf{x}, 0)
$$

Suppose that two solutions $\left(\mathbf{u}_{1}, \theta_{1}, \mathbf{p}_{1}\right)$ and $\left(\mathbf{u}_{2}, \theta_{2}, \mathbf{p}_{2}\right)$ to (1)-(3) exist. Subtract, equation by equation, the variational formulation (7)-(8) corresponding to solution $\left(\mathbf{u}_{2}, \theta_{2}, \mathbf{p}_{2}\right)$ from that one corresponding to solution $\left(\mathbf{u}_{1}, \theta_{1}, \mathbf{p}_{1}\right)$. Set $\mathbf{u}=\mathbf{u}_{1}-\mathbf{u}_{2}, \mathbf{p}=\mathbf{p}_{1}-\mathbf{p}_{2}$ and $\theta=\theta_{1}-\theta_{2}$. Then $\mathbf{u}(\mathbf{x}, 0)=\mathbf{0}, \mathbf{u}(\mathbf{x}, T)=\mathbf{0}$, $\partial_{t} \mathbf{u}(\mathbf{x}, 0)=\mathbf{0}$ and $\theta(\mathbf{x}, 0)=0$. This implies that the integrals in (9) and $(10)$ are equal to zero. We obtain

$$
\left(\varrho \partial_{t t} \mathbf{u}(t), \boldsymbol{\varphi}\right)+\left(\mathbf{g}\left(\partial_{t} \mathbf{u}_{\mathbf{1}}(t)\right)-\mathbf{g}\left(\partial_{t} \mathbf{u}_{\mathbf{2}}(t)\right), \boldsymbol{\varphi}\right)+\left(\mathcal{L}^{\mathrm{e}} \mathbf{u}(t), \boldsymbol{\varphi}\right)+(\mathbb{B} \nabla \theta(t), \boldsymbol{\varphi})=(\mathbf{p}, \boldsymbol{\varphi})
$$

and

$$
\left(\varrho C_{s} \partial_{t} \theta(t), \psi\right)+(\mathbb{K} \nabla \theta(t), \nabla \psi)+((K * \nabla \theta)(t), \nabla \psi)-\left(T_{0} \mathbb{B} \partial_{t} \mathbf{u}(t), \nabla \psi\right)=0,
$$

for all $\boldsymbol{\varphi} \in \mathbf{H}_{0}^{1}(\Omega)$ and $\psi \in \mathrm{H}_{0}^{1}(\Omega)$. First, we prove that $\mathbf{u}=\mathbf{0}$ and $\theta=0$ and then show that $\mathbf{p}=\mathbf{0}$. 
In the first part of the proof, we want to eliminate $\mathbf{p}$. This can be done by setting $\varphi=\partial_{t} \mathbf{u}(t)$ in (11) and integrating in time over $(0, T)$. Using (9) and (10), we obtain that equation (11) reduces to

$$
\begin{aligned}
\frac{\left\|\sqrt{\varrho} \partial_{t} \mathbf{u}(T)\right\|^{2}}{2}+\int_{0}^{T}\left(\mathbf{g}\left(\partial_{t} \mathbf{u}_{\mathbf{1}}(t)\right)-\mathbf{g}\left(\partial_{t} \mathbf{u}_{\mathbf{2}}(t)\right), \partial_{t} \mathbf{u}_{1}(t)-\partial_{t} \mathbf{u}_{2}(t)\right) \mathrm{d} t & \\
& +\int_{0}^{T}\left(\mathbb{B} \nabla \theta(t), \partial_{t} \mathbf{u}(t)\right) \mathrm{d} t=0
\end{aligned}
$$

since $\mathbf{u}(\mathbf{x}, 0)=\mathbf{u}(\mathbf{x}, T)=\partial_{t} \mathbf{u}(\mathbf{x}, 0)=\mathbf{0}$. Then, taking $\psi=\frac{\theta(t)}{T_{0}}$ in 12 and applying Green's theorem, one obtains

$$
\begin{aligned}
\frac{\left\|\sqrt{\varrho C_{s}} \theta(T)\right\|^{2}}{2 T_{0}}+\frac{1}{T_{0}} \int_{0}^{T}(\mathbb{K} \nabla \theta(t), \nabla \theta(t)) \mathrm{d} t+\frac{1}{T_{0}} \int_{0}^{T}((K * \nabla \theta)(t), \nabla \theta(t)) \mathrm{d} t & -\int_{0}^{T}\left(\mathbb{B} \partial_{t} \mathbf{u}(t), \nabla \theta(t)\right) \mathrm{d} t=0
\end{aligned}
$$

since $\theta(\mathbf{x}, 0)=0$. Moreover, the symmetry of $\mathbb{B}$ implies that

$$
\int_{0}^{T}\left(\mathbb{B} \nabla \theta(t), \partial_{t} \mathbf{u}(t)\right) \mathrm{d} t=\int_{0}^{T}\left(\nabla \theta(t), \mathbb{B} \partial_{t} \mathbf{u}(t)\right) \mathrm{d} t .
$$

By adding (13) and (14) and, at the same time, accounting for the relation above, it follows that the coupling terms are cancelled out, i.e.

$$
\begin{aligned}
& \frac{\left\|\sqrt{\varrho} \partial_{t} \mathbf{u}(T)\right\|^{2}}{2}+\int_{0}^{T}\left(\mathbf{g}\left(\partial_{t} \mathbf{u}_{\mathbf{1}}(t)\right)-\mathbf{g}\left(\partial_{t} \mathbf{u}_{\mathbf{2}}(t)\right), \partial_{t} \mathbf{u}_{1}(t)-\partial_{t} \mathbf{u}_{2}(t)\right) \mathrm{d} t \\
& +\frac{\left\|\sqrt{\varrho C_{s}} \theta(T)\right\|^{2}}{2 T_{0}}+\frac{1}{T_{0}} \int_{0}^{T}(\mathbb{K} \nabla \theta(t), \nabla \theta(t)) \mathrm{d} t+\frac{1}{T_{0}} \int_{0}^{T}((K * \nabla \theta)(t), \nabla \theta(t)) \mathrm{d} t=0 .
\end{aligned}
$$

The positive semidefiniteness of $K$ and the positive definiteness of $\mathbb{K}$ imply that

$$
\int_{0}^{T}((K * \nabla \theta)(t), \nabla \theta(t)) \mathrm{d} t \geqslant 0 \quad \text { and } \quad \int_{0}^{T}(\mathbb{K} \nabla \theta(t), \nabla \theta(t)) \mathrm{d} t \geqslant 0
$$

respectively. Thus from (15) it follows that

$$
\int_{0}^{T}\left(\mathbf{g}\left(\partial_{t} \mathbf{u}_{\mathbf{1}}(t)\right)-\mathbf{g}\left(\partial_{t} \mathbf{u}_{\mathbf{2}}(t)\right), \partial_{t} \mathbf{u}_{1}(t)-\partial_{t} \mathbf{u}_{2}(t)\right) \mathrm{d} t=0 .
$$

Here, we can also see the reason why the damping term is necessary. Without this term, one would have no guarantee that $\mathbf{u}=\mathbf{0}$ or $\theta=0$. Employing the fact that the vector field $\mathbf{g}$ is componentwise strictly monotonically increasing, we obtain that $\partial_{t} \mathbf{u}_{1}=\partial_{t} \mathbf{u}_{2}$ or $\partial_{t} \mathbf{u}=\mathbf{0}$, i.e. $\mathbf{u}$ is constant in time. Therefore, it follows that

$$
\mathbf{u}(\mathbf{x}, 0)=\mathbf{0} \Rightarrow \mathbf{u}(\mathbf{x}, t)=\mathbf{0} \text { for a.a. }(\mathbf{x}, t) \in Q_{T}
$$

and this implies that equation 12 reduces to

$$
\left(\varrho C_{s} \partial_{t} \theta(t), \psi\right)+(\mathbb{K} \nabla \theta(t), \nabla \psi)+((K * \nabla \theta)(t), \nabla \psi)=0 .
$$


Taking $\psi=\theta(t)$ in the relation above and integrating it with respect to time over $(0, \eta) \subset(0, T)$ yields

$$
\theta=0 \text { a.e. in } Q_{T} \text {. }
$$

Finally, substituting $\mathbf{u}=\mathbf{0}$ and $\theta=0$ in (11) gives

$$
(\mathbf{p}, \boldsymbol{\varphi})=0, \quad \forall \boldsymbol{\varphi} \in \mathbf{H}_{0}^{1}(\Omega),
$$

which implies that $\mathbf{p}=\mathbf{0}$ in $\mathbf{L}^{2}(\Omega)$.

Remark 3.1. An example for the convolution kernel $K$ is given by $K(t)=t^{-\alpha}, t \in(0, T]$, with $0<\alpha<1$. Another example that fits in this setting is $K(t)=\exp (-t), t \in[0, T]$.

Theorem 3.2 (Uniqueness for $K \in \mathrm{L}^{1}(0, T)$ ). Suppose that

$$
K \in \mathrm{L}^{1}(0, T) \quad \text { s.t. } \quad \int_{0}^{T}|K(t)| \mathrm{d} t \leqslant k_{0} .
$$

Then, there exists at most one $\mathbf{p} \in \mathbf{L}^{2}(\Omega)$ such that problem (1) and condition (3) hold.

Proof. The proof follows the ideas of Theorem 3.1. We only point out the differences.

Analogous to Theorem 3.1, one arrives at relation (15). The positive definiteness of $\mathbb{K}$ implies that

$$
\begin{aligned}
& \frac{\left\|\sqrt{\varrho} \partial_{t} \mathbf{u}(T)\right\|^{2}}{2}+\int_{0}^{T}\left(\mathbf{g}\left(\partial_{t} \mathbf{u}_{\mathbf{1}}(t)\right)-\mathbf{g}\left(\partial_{t} \mathbf{u}_{2}(t)\right), \partial_{t} \mathbf{u}_{1}(t)-\partial_{t} \mathbf{u}_{2}(t)\right) \mathrm{d} t \\
& +\frac{\left\|\sqrt{\varrho C_{s}} \theta(T)\right\|^{2}}{2 T_{0}}+\frac{k_{0}}{T_{0}} \int_{0}^{T}\|\nabla \theta(t)\|^{2} \mathrm{~d} t \leqslant\left|\frac{1}{T_{0}} \int_{0}^{T}((K * \nabla \theta)(t), \nabla \theta(t)) \mathrm{d} t\right| .
\end{aligned}
$$

We further use Young's inequality for convolutions in this proof, namely

$$
\|f * g\|_{r} \leqslant\|f\|_{p}\|g\|_{q}, \quad \frac{1}{p}+\frac{1}{q}=\frac{1}{r}+1, \quad 1 \leqslant p, q, r \leqslant \infty .
$$

By applying Young's inequality (17) to the right-hand side of relation (16), one obtains

$$
\begin{aligned}
& \left|\int_{0}^{T}((K * \nabla \theta)(t), \nabla \theta(t)) \mathrm{d} t\right|=\left|\int_{\Omega} \int_{0}^{T}(K * \nabla \theta)(\mathbf{x}, t) \nabla \theta(\mathbf{x}, t) \mathrm{d} t \mathrm{~d} \mathbf{x}\right| \\
& \leqslant \int_{\Omega}\left|\int_{0}^{T}(K * \nabla \theta)(\mathbf{x}, t) \nabla \theta(\mathbf{x}, t) \mathrm{d} t\right| \mathrm{d} \mathbf{x} \\
& \leqslant \int_{\Omega} \sqrt{\int_{0}^{T}(K * \nabla \theta)^{2}(\mathbf{x}, t) \mathrm{d} t} \sqrt{\int_{0}^{T} \nabla \theta(\mathbf{x}, t)^{2} \mathrm{~d} t} \mathrm{~d} \mathbf{x} \\
& \stackrel{[177}{\leqslant} \int_{\Omega}\left(\int_{0}^{T}|K(t)| \mathrm{d} t\right) \sqrt{\int_{0}^{T} \nabla \theta(\mathbf{x}, t)^{2} \mathrm{~d} t} \sqrt{\int_{0}^{T} \nabla \theta(\mathbf{x}, t)^{2} \mathrm{~d} t} \mathrm{~d} \mathbf{x} \\
& \leqslant\left(\int_{0}^{T}|K(t)| \mathrm{d} t\right) \int_{0}^{T}\|\nabla \theta(t)\|^{2} \mathrm{~d} t,
\end{aligned}
$$

and hence equation (16) reduces to

$$
\int_{0}^{T}\left(\mathbf{g}\left(\partial_{t} \mathbf{u}_{\mathbf{1}}(t)\right)-\mathbf{g}\left(\partial_{t} \mathbf{u}_{\mathbf{2}}(t)\right), \partial_{t} \mathbf{u}_{1}(t)-\partial_{t} \mathbf{u}_{2}(t)\right) \mathrm{d} t=0 .
$$

Therefore, $\mathbf{u}=\mathbf{0}$ in $\mathbf{L}^{2}(\Omega)$. Then, it follows that $\theta=0$ in $\mathrm{L}^{2}(\Omega)$ and $\mathbf{p}=\mathbf{0}$ in $\mathbf{L}^{2}(\Omega)$. 
Theorem 3.3 (Uniqueness for a bounded convolution kernel). Suppose that

$$
\exists C>0 \quad \text { s.t. } \max _{t \in[0, T]}|K(t)| \leqslant C .
$$

Then, there exists at most $\mathbf{p} \in \mathbf{L}^{2}(\Omega)$ such that problem (1) and condition (3) hold.

Proof. The proof follows again the same lines of those for Theorems 3.1 and 3.2 and, analogously, one arrives at (16). By using Young's and Hölder's inequalities, the term in the right-hand side of equation (16) can be estimated by

$$
\begin{aligned}
& \left|\int_{0}^{T}\left(\int_{0}^{t} K(t-s) \nabla \theta(s) \mathrm{d} s, \nabla \theta(t)\right) \mathrm{d} t\right| \leqslant C_{\varepsilon} \int_{0}^{T}\left\|\int_{0}^{t} K(t-s) \nabla \theta(s) \mathrm{d} s\right\|^{2} \mathrm{~d} t+\varepsilon \int_{0}^{T}\|\nabla \theta(t)\|^{2} \mathrm{~d} t \\
& \leqslant C_{\varepsilon} \int_{0}^{T}\left(\int_{0}^{t}|K(t-s)|\|\nabla \theta(s)\| \mathrm{d} s\right)^{2} \mathrm{~d} t+\varepsilon \int_{0}^{T}\|\nabla \theta(t)\|^{2} \mathrm{~d} t \\
& \leqslant C_{\varepsilon} \int_{0}^{T}\left(\int_{0}^{t}|K(t-s)|^{2} \mathrm{~d} s\right)\left(\int_{0}^{t}\|\nabla \theta(s)\|^{2} \mathrm{~d} s\right) \mathrm{d} t+\varepsilon \int_{0}^{T}\|\nabla \theta(t)\|^{2} \mathrm{~d} t \\
& \leqslant C_{\varepsilon} \int_{0}^{T}\left(\int_{0}^{t}\|\nabla \theta(s)\|^{2} \mathrm{~d} s\right) \mathrm{d} t+\varepsilon \int_{0}^{T}\|\nabla \theta(t)\|^{2} \mathrm{~d} t .
\end{aligned}
$$

Fixing $\varepsilon$ sufficiently small and applying Grönwall's lemma implies that $\mathbf{u}=\mathbf{p}=\mathbf{0}$ and $\theta=0$.

Remark 3.2. The exponentially decaying kernel $K(t)=\exp (-t), t \in[0, T]$, satisfies the requirement of Theorem 3.3

\section{Reconstruction of the vector source in a linear case}

In the following, it is assumed that $\mathbf{g}$ is linear, i.e. without any loss of the generality

$$
\mathbf{g}=g \mathbf{I} \quad \text { with } \quad g_{1} \geqslant g(\mathbf{x}) \geqslant g_{0}>0 \text { for a.a. } \mathbf{x} \in \Omega .
$$

It is possible to prove the convergence of the proposed numerical scheme only in this situation. First, the well-posedness of problem (1) for a given source $\mathbf{p}$ is investigated. In this case, the variational problem (7)-(8) reduces to:

Given $\mathbf{r}:(0, T] \rightarrow \mathbf{L}^{2}(\Omega)$ in $\mathrm{L}^{2}\left((0, T), \mathbf{L}^{2}(\Omega)\right), h:(0, T] \rightarrow \mathrm{L}^{2}(\Omega)$ in $\mathrm{L}^{2}\left((0, T), \mathrm{L}^{2}(\Omega)\right), \overline{\mathbf{u}}_{0}(\mathbf{x}) \in$ $\mathbf{H}^{1}(\Omega), \overline{\mathbf{u}}_{1}(\mathbf{x}) \in \mathbf{L}^{2}(\Omega), \bar{\theta}_{0} \in \mathrm{H}^{1}(\Omega)$ and $\boldsymbol{\xi}_{T} \in \mathbf{L}^{2}(\Omega)$, find $(\mathbf{u}(t), \theta(t)) \in \mathbf{H}_{0}^{1}(\Omega) \times \mathrm{H}_{0}^{1}(\Omega)$, with $\partial_{t} \mathbf{u}(t) \in \mathbf{L}^{2}(\Omega), \partial_{t t} \mathbf{u}(t) \in \mathbf{H}_{0}^{1}(\Omega)^{*}$ and $\partial_{t} \theta(t) \in \mathrm{H}_{0}^{1}(\Omega)^{*}$, such that

$$
\begin{aligned}
& \left(\varrho \partial_{t t} \mathbf{u}(t), \boldsymbol{\varphi}\right)+\left(\mathbf{g}\left(\partial_{t} \mathbf{u}(t)\right), \boldsymbol{\varphi}\right)+\left(\mathcal{L}^{\mathrm{e}} \mathbf{u}(t), \boldsymbol{\varphi}\right)+(\mathbb{B} \nabla \theta(t), \boldsymbol{\varphi})=(\mathbf{r}(t), \boldsymbol{\varphi}) \\
& \left(\varrho C_{s} \partial_{t} \theta(t), \psi\right)+(\mathbb{K} \nabla \theta(t), \nabla \psi)+((K * \nabla \theta)(t), \nabla \psi)-T_{0}\left(\mathbb{B} \partial_{t} \mathbf{u}(t), \nabla \psi\right)=(h(t), \psi)
\end{aligned}
$$

for all $\boldsymbol{\varphi} \in \mathbf{H}_{0}^{1}(\Omega)$ and $\psi \in \mathrm{H}_{0}^{1}(\Omega)$ and a.a. $t \in[0, T]$.

Theorem 4.1 (Well-posedness of the direct problem). Assume that $\mathbf{r}:(0, T] \rightarrow \mathbf{L}^{2}(\Omega)$ and $h:(0, T] \rightarrow$ $\mathrm{L}^{2}(\Omega)$ belong to $\mathrm{L}^{2}\left((0, T), \mathbf{L}^{2}(\Omega)\right)$ and $\mathrm{L}^{2}\left((0, T), \mathrm{L}^{2}(\Omega)\right)$, respectively, $\overline{\mathbf{u}}_{0}(\mathbf{x}) \in \mathbf{H}^{1}(\Omega), \overline{\mathbf{u}}_{1}(\mathbf{x}) \in \mathbf{L}^{2}(\Omega)$ and $\bar{\theta}_{0} \in \mathrm{H}^{1}(\Omega)$. Assume that any of the following conditions holds for the kernel $K:(0, T] \rightarrow \mathbb{R}$ : 
(i) $K$ is strongly positive definite, i.e. $K^{\prime}(t) \not \equiv 0$ and $(-1)^{j} K^{(j)}(t) \geqslant 0, t>0, j=0,1,2$;

(ii) $K \in \mathrm{L}^{1}(0, T) \quad$ s.t. $\quad \int_{0}^{T}|K(t)| \mathrm{d} t \leqslant k_{0}$;

(iii) $\exists C>0 \quad$ s.t. $\max _{t \in[0, T]}|K(t)| \leqslant C$.

Then, the variational problem $(18)-(19)$ has a unique solution $(\mathbf{u}, \theta)$ such that $\mathbf{u} \in \mathbf{C}\left([0, T], \mathbf{L}^{2}(\Omega)\right) \cap$ $\mathrm{L}^{2}\left((0, T), \mathbf{H}_{0}^{1}(\Omega)\right), \partial_{t} \mathbf{u} \in \mathrm{C}\left([0, T], \mathbf{L}^{2}(\Omega)\right), \partial_{t t} \mathbf{u} \in \mathrm{L}^{2}\left((0, T), \mathbf{H}_{0}^{1}(\Omega)^{*}\right), \theta \in \mathrm{C}\left([0, T], \mathbf{L}^{2}(\Omega)\right) \cap$ $\mathrm{L}^{2}\left((0, T), \mathrm{H}_{0}^{1}(\Omega)\right)$ and $\partial_{t} \theta \in \mathrm{L}^{2}\left((0, T), \mathrm{H}_{0}^{1}(\Omega)^{*}\right)$.

Moreover, when $\overline{\mathbf{u}}_{0}(\mathbf{x})=\mathbf{0}, \overline{\mathbf{u}}_{1}(\mathbf{x})=\mathbf{0}, \bar{\theta}_{0}=0, h=0$ and $\mathbf{r}=\mathbf{r}(\mathbf{x})$, the following estimate holds

$$
\max _{t \in[0, T]}\left\{\left\|\partial_{t} \mathbf{u}(t)\right\|^{2}+\|\mathbf{u}(t)\|_{\mathbf{H}_{0}^{1}(\Omega)}^{2}+\|\theta(t)\|^{2}\right\}+\int_{0}^{T}\|\nabla \theta(t)\|^{2} \mathrm{~d} t \leqslant C\|\mathbf{r}\|^{2} .
$$

Proof. (i) It is assumed that the kernel $K$ is strongly positive definite. To address the existence of a solution to the variational problem (18)-(19), the semidiscretization in time is employed and this is based on Rothe's method [22]. The interval $[0, T]$ is divided into $n \in \mathbb{N}$ equidistant subintervals $\left[t_{i-1}, t_{i}\right]$ with the time step $\tau=\frac{T}{n}<1$, thus $t_{i}=i \tau, i=0, \ldots, n$. With the standard notation for the discretized fields, for any function $z$

$$
z_{i} \approx z\left(t_{i}\right), \quad \partial_{t} z(t) \approx \delta z_{i}=\frac{z_{i}-z_{i-1}}{\tau} \quad \text { and } \quad \partial_{t t} z(t) \approx \delta^{2} z_{i}=\frac{z_{i}-z_{i-1}}{\tau^{2}}-\frac{\delta z_{i-1}}{\tau},
$$

the following linear recurrent scheme is proposed to approximate the original problem (11)-(12) for $i=$ $1, \ldots, n\left(\varphi \in \mathbf{H}_{0}^{1}(\Omega)\right.$ and $\left.\psi \in \mathrm{H}_{0}^{1}(\Omega)\right)$ :

$$
\left(\varrho \delta^{2} \mathbf{u}_{i}, \boldsymbol{\varphi}\right)+\left(g \delta \mathbf{u}_{i}, \boldsymbol{\varphi}\right)+\left(\mathcal{L}^{\mathrm{e}} \mathbf{u}_{i}, \boldsymbol{\varphi}\right)+\left(\mathbb{B} \nabla \theta_{i}, \boldsymbol{\varphi}\right)=\left(\mathbf{r}_{i}, \boldsymbol{\varphi}\right)
$$

and

$$
\left(\varrho C_{s} \delta \theta_{i}, \psi\right)+\left(\mathbb{K} \nabla \theta_{i}, \nabla \psi\right)+\left(\sum_{l=1}^{i} K_{l} \nabla \theta_{i-l} \tau, \nabla \psi\right)-T_{0}\left(\mathbb{B} \delta \mathbf{u}_{i}, \nabla \psi\right)=\left(h_{i}, \psi\right) .
$$

This is equivalent to solving

$$
a\left(\left(\begin{array}{c}
\mathbf{u}_{i} \\
\theta_{i}
\end{array}\right),\left(\begin{array}{c}
\varphi \\
\psi
\end{array}\right)\right)=F_{i}\left(\begin{array}{c}
\varphi \\
\psi
\end{array}\right), \quad \mathbf{u}_{0}=\overline{\mathbf{u}}_{0}, \quad \delta \mathbf{u}_{0}=\overline{\mathbf{u}}_{1}, \quad \theta_{0}=\bar{\theta}_{0}
$$

for any $1 \leq i \leq n$, where $a:\left(\mathbf{H}_{0}^{1}(\Omega) \times \mathrm{H}_{0}^{1}(\Omega)\right)^{2} \rightarrow \mathbb{R}$ and $F_{i}: \mathbf{H}_{0}^{1}(\Omega) \times \mathrm{H}_{0}^{1}(\Omega) \rightarrow \mathbb{R}$ are defined as

$$
a\left(\left(\begin{array}{c}
\mathbf{u}_{i} \\
\theta_{i}
\end{array}\right),\left(\begin{array}{c}
\boldsymbol{\varphi} \\
\psi
\end{array}\right)\right):=\frac{T_{0}}{\tau} L_{1}+L_{2}, \quad F_{i}\left(\begin{array}{l}
\boldsymbol{\varphi} \\
\psi
\end{array}\right):=\frac{T_{0}}{\tau} R_{1}+R_{2},
$$

with

$$
\begin{aligned}
L_{1} & :=\frac{1}{\tau^{2}}\left(\varrho \mathbf{u}_{i}, \boldsymbol{\varphi}\right)+\frac{1}{\tau}\left(g \mathbf{u}_{i}, \boldsymbol{\varphi}\right)+\left(\mathcal{L}^{\mathrm{e}} \mathbf{u}_{i}, \boldsymbol{\varphi}\right)+\left(\mathbb{B} \nabla \theta_{i}, \boldsymbol{\varphi}\right) \\
& =\left(\mathbf{r}_{i}, \boldsymbol{\varphi}\right)+\frac{1}{\tau^{2}}\left(\varrho \mathbf{u}_{i-1}, \boldsymbol{\varphi}\right)+\frac{1}{\tau}\left(g \mathbf{u}_{i-1}, \boldsymbol{\varphi}\right)+\frac{1}{\tau}\left(\varrho \delta \mathbf{u}_{i-1}, \boldsymbol{\varphi}\right)=: R_{1},
\end{aligned}
$$


Therefore, one obtains

$$
\begin{aligned}
& 2 \sum_{i=1}^{j}\left(\mathcal{L}^{\mathrm{e}} \mathbf{u}_{i}, \delta \mathbf{u}_{i}\right) \tau=2 \sum_{i=1}^{j} a\left(\mathbf{u}_{i}, \delta \mathbf{u}_{i}\right) \tau=a\left(\mathbf{u}_{j}, \mathbf{u}_{j}\right)-a\left(\overline{\mathbf{u}}_{0}, \overline{\mathbf{u}}_{0}\right)+\sum_{i=1}^{j} a\left(\mathbf{u}_{i}-\mathbf{u}_{i-1}, \mathbf{u}_{i}-\mathbf{u}_{i-1}\right) \\
& =\left(\mathcal{L}^{\mathrm{e}} \mathbf{u}_{j}, \mathbf{u}_{j}\right)-\left(\mathcal{L}^{\mathrm{e}} \overline{\mathbf{u}}_{0}, \overline{\mathbf{u}}_{0}\right)+\sum_{i=1}^{j}\left(\mathcal{L}^{\mathrm{e}}\left(\mathbf{u}_{i}-\mathbf{u}_{i-1}\right), \mathbf{u}_{i}-\mathbf{u}_{i-1}\right) .
\end{aligned}
$$

The bilinear form $a$ is coercive and continuous on $\mathbf{H}_{0}^{1}(\Omega) \times \mathrm{H}_{0}^{1}(\Omega)$. Moreover, $F_{i} \in\left(\mathbf{H}_{0}^{1}(\Omega) \times \mathrm{H}_{0}^{1}(\Omega)\right)^{*}$ if $\mathbf{u}_{i-1}, \delta \mathbf{u}_{i-1} \in \mathbf{L}^{2}(\Omega), \theta_{i-1} \in \mathrm{L}^{2}(\Omega)$ and $\nabla \theta_{l} \in \mathrm{L}^{2}(\Omega)$, for $0 \leq l \leq i-1$. For every $1 \leq i \leq n$, the Lax-Milgram lemma gives the existence and uniqueness of a solution $\left(\mathbf{u}_{i}, \theta_{i}\right) \in \mathbf{H}_{0}^{1}(\Omega) \times \mathrm{H}_{0}^{1}(\Omega)$ to 23)-24) or, equivalently, 21)-22), if $\overline{\mathbf{u}}_{0}, \overline{\mathbf{u}}_{1} \in \mathbf{L}^{2}(\Omega)$ and $\bar{\theta}_{0} \in \mathrm{H}^{1}(\Omega)$.

Next, a priori estimates are derived. These estimates serve as uniform bounds to prove the convergence of the semidiscrete scheme (21)-(22).

We set $\varphi=\delta \mathbf{u}_{i} \tau$ and $\psi=\frac{\theta_{i}}{T_{0}} \tau$ in (21)-(22) and sum up these equations for $1 \leq i \leq j$, with $1 \leqslant j \leqslant n$. Then, we add both resulting equations, hence the coupling term cancels out and one obtains

$$
\begin{aligned}
& \sum_{i=1}^{j}\left(\varrho \delta^{2} \mathbf{u}_{i}, \delta \mathbf{u}_{i}\right) \tau+\sum_{i=1}^{j}\left\|\sqrt{g} \delta \mathbf{u}_{i}\right\|^{2} \tau+\sum_{i=1}^{j}\left(\mathcal{L}^{\mathrm{e}} \mathbf{u}_{i}, \delta \mathbf{u}_{i}\right) \tau+\frac{1}{T_{0}} \sum_{i=1}^{j}\left(\varrho C_{s} \delta \theta_{i}, \theta_{i}\right) \tau \\
& +\frac{1}{T_{0}} \sum_{i=1}^{j}\left(\mathbb{K} \nabla \theta_{i}, \nabla \theta_{i}\right) \tau+\frac{1}{T_{0}} \sum_{i=1}^{j}\left(\sum_{l=1}^{i} K_{l} \nabla \theta_{i-l} \tau, \nabla \theta_{i}\right) \tau=\sum_{i=1}^{j}\left(\mathbf{r}_{i}, \delta \mathbf{u}_{i}\right) \tau+\frac{1}{T_{0}} \sum_{i=1}^{j}\left(h_{i}, \theta_{i}\right) \tau .
\end{aligned}
$$

We use Abel's summation rule for three terms on the left-hand side, namely

$$
\begin{aligned}
& 2 \sum_{i=1}^{j}\left(\varrho \delta^{2} \mathbf{u}_{i}, \delta \mathbf{u}_{i}\right) \tau=\left\|\sqrt{\varrho} \delta \mathbf{u}_{j}\right\|^{2}-\left\|\sqrt{\varrho} \overline{\mathbf{u}}_{1}\right\|^{2}+\sum_{i=1}^{j}\left\|\sqrt{\varrho} \delta \mathbf{u}_{i}-\sqrt{\varrho} \delta \mathbf{u}_{i-1}\right\|^{2}, \\
& 2 \sum_{i=1}^{j}\left(\varrho C_{s} \delta \theta_{i}, \theta_{i}\right) \tau=\left\|\sqrt{\varrho C_{s}} \theta_{j}\right\|^{2}-\left\|\sqrt{\varrho C_{s}} \bar{\theta}_{0}\right\|^{2}+\sum_{i=1}^{j}\left\|\sqrt{\varrho C_{s}} \theta_{i}-\sqrt{\varrho C_{s}} \theta_{i-1}\right\|^{2} .
\end{aligned}
$$

Note that by the symmetry of $a$, the following relation holds

$$
\delta a\left(\mathbf{u}_{i}, \mathbf{u}_{i}\right)=a\left(\delta \mathbf{u}_{i}, \mathbf{u}_{i}\right)+a\left(\mathbf{u}_{i-1}, \delta \mathbf{u}_{i}\right)=2 a\left(\mathbf{u}_{i}, \delta \mathbf{u}_{i}\right)-a\left(\delta \mathbf{u}_{i} \tau, \delta \mathbf{u}_{i}\right) .
$$


By the ellipticity of $\mathcal{L}^{\mathrm{e}}$, it follows that

$$
\left(\mathcal{L}^{\mathrm{e}} \mathbf{u}_{j}, \mathbf{u}_{j}\right) \geqslant m\left\|\mathbf{u}_{j}\right\|_{\mathbf{H}_{0}^{1}(\Omega)}^{2}
$$

The thermal conductivity tensor $\mathbb{K}$ is positive definite, i.e.

$$
\sum_{i=1}^{j}\left(\mathbb{K} \nabla \theta_{i}, \nabla \theta_{i}\right) \tau \geqslant k_{0} \sum_{i=1}^{j}\left\|\nabla \theta_{i}\right\|^{2} \tau
$$

The strongly positive definiteness of $K$ implies that

$$
\sum_{i=1}^{j}\left(\sum_{l=1}^{i} K_{l} \nabla \theta_{i-l} \tau, \nabla \theta_{i}\right) \tau \geqslant 0
$$

The first and second term in the right-hand side of the above relationship can be estimated, in a classical way, by using Cauchy's and Young's inequalities, i.e.

$$
\left|\sum_{i=1}^{j}\left(\mathbf{r}_{i}, \delta \mathbf{u}_{i}\right) \tau\right| \leqslant C_{\varepsilon}+\varepsilon \sum_{i=1}^{j}\left\|\delta \mathbf{u}_{i}\right\|^{2} \tau \quad \text { and } \quad\left|\sum_{i=1}^{j}\left(h_{i}, \theta_{i}\right) \tau\right| \leqslant C_{\varepsilon}+\varepsilon \sum_{i=1}^{j}\left\|\theta_{i}\right\|^{2} \tau .
$$

Collecting all the results above, employing the $\mathbf{H}_{0}^{1}(\Omega)$-ellipticity of $\mathcal{L}^{\mathrm{e}}$ and fixing $\varepsilon$ small enough yield

$$
\begin{aligned}
& \max _{1 \leqslant j \leqslant n}\left\|\delta \mathbf{u}_{j}\right\|^{2}+\sum_{i=1}^{n}\left\|\delta \mathbf{u}_{i}-\delta \mathbf{u}_{i-1}\right\|^{2}+\sum_{i=1}^{n}\left\|\delta \mathbf{u}_{i}\right\|^{2} \tau+\max _{1 \leqslant j \leqslant n}\left\|\mathbf{u}_{j}\right\|_{\mathbf{H}_{0}^{1}(\Omega)}^{2} \\
& +\sum_{i=1}^{n}\left\|\mathbf{u}_{i}-\mathbf{u}_{i-1}\right\|_{\mathbf{H}_{0}^{1}(\Omega)}^{2}+\max _{1 \leqslant j \leqslant n}\left\|\theta_{j}\right\|^{2}+\sum_{i=1}^{n}\left\|\theta_{i}-\theta_{i-1}\right\|^{2}+\sum_{i=1}^{n}\left\|\nabla \theta_{i}\right\|^{2} \tau \leqslant C,
\end{aligned}
$$

with $C=C\left(\left\|\overline{\mathbf{u}}_{0}\right\|_{\mathbf{H}_{0}^{1}(\Omega)},\left\|\overline{\mathbf{u}}_{1}\right\|,\left\|\bar{\theta}_{0}\right\|_{\mathrm{H}^{1}(\Omega)},\|\mathbf{r}\|_{\mathrm{L}^{2}\left((0, T), \mathbf{L}^{2}(\Omega)\right)},\|h\|_{\mathrm{L}^{2}\left((0, T), \mathrm{L}^{2}(\Omega)\right)}\right)$. From this estimate 25), it follows that

$$
\sum_{i=1}^{n}\left\|\delta^{2} \mathbf{u}_{i}\right\|_{\mathbf{H}_{0}^{1}(\Omega)^{*}}^{2} \tau \leqslant C \quad \text { and } \quad \sum_{i=1}^{n}\left\|\delta \theta_{i}\right\|_{\mathrm{H}_{0}^{1}(\Omega)^{*}}^{2} \tau \leqslant C .
$$

We further introduce the following piecewise linear in time functions $\mathbf{u}_{n}:[0, T] \rightarrow \mathrm{L}^{2}(\Omega)$ and $\mathbf{v}_{n}:[0, T] \rightarrow$ $\mathrm{L}^{2}(\Omega)$

$$
\begin{aligned}
& \mathbf{u}_{n}(0)=\overline{\mathbf{u}}_{0} \\
& \mathbf{u}_{n}(t)=\mathbf{u}_{i-1}+\left(t-t_{i-1}\right) \delta \mathbf{u}_{i}, \quad t \in\left(t_{i-1}, t_{i}\right], \quad 1 \leq i \leq n \\
& \mathbf{v}_{n}(0)=\overline{\mathbf{u}}_{1} \\
& \mathbf{v}_{n}(t)=\delta \mathbf{u}_{i-1}+\left(t-t_{i-1}\right) \delta^{2} \mathbf{u}_{i} \quad t \in\left(t_{i-1}, t_{i}\right], \quad 1 \leq i \leq n
\end{aligned}
$$

and the piecewise constant in time functions $\overline{\mathbf{u}}_{n}:[0, T] \rightarrow \mathrm{L}^{2}(\Omega)$ and $\overline{\mathbf{v}}_{n}:[0, T] \rightarrow \mathrm{L}^{2}(\Omega)$

$$
\begin{array}{llll}
\overline{\mathbf{u}}_{n}(0)=\overline{\mathbf{u}}_{0}, & \overline{\mathbf{u}}_{n}(t)=\mathbf{u}_{i}, & t \in\left(t_{i-1}, t_{i}\right], & 1 \leq i \leq n \\
\overline{\mathbf{v}}_{n}(0)=\overline{\mathbf{u}}_{1}, & \overline{\mathbf{v}}_{n}(t)=\delta \mathbf{u}_{i}, & t \in\left(t_{i-1}, t_{i}\right], & 1 \leq i \leq n .
\end{array}
$$


Analogously, we define the following functions $\theta_{n}, \bar{\theta}_{n}, \bar{K}_{n}, \overline{\mathbf{r}}_{n}$ and $\bar{h}_{n}$, respectively, and note that $\overline{\mathbf{v}}_{n}=$ $\partial_{t} \mathbf{u}_{n}$. We also introduce the notation $\lceil\cdot\rceil_{\tau}$ defined as $\lceil t\rceil_{\tau}=i$, for $t \in\left(t_{i-1}, t_{i}\right]$. Using these so-called Rothe's functions, the variational formulation (21)-(22) can be rewritten, for a.a. $t \in[0, T]$, as

$$
\begin{aligned}
& \left(\rho \partial_{t} \mathbf{v}_{n}(t), \boldsymbol{\varphi}\right)+\left(g \partial_{t} \mathbf{u}_{n}(t), \boldsymbol{\varphi}\right)+\left(\mathcal{L}^{\mathrm{e}} \overline{\mathbf{u}}_{n}(t), \boldsymbol{\varphi}\right)+\left(\mathbb{B} \nabla \bar{\theta}_{n}(t), \boldsymbol{\varphi}\right)=\left(\overline{\mathbf{r}}_{n}(t), \boldsymbol{\varphi}\right) \\
& \left(\varrho C_{s} \partial_{t} \theta_{n}(t), \psi\right)+\left(\mathbb{K} \nabla \bar{\theta}_{n}(t), \nabla \psi\right)+\sum_{l=1}^{\lceil t\rceil_{\tau}} \bar{K}_{n}\left(t_{l}\right)\left(\nabla \bar{\theta}_{n}\left(t-t_{l}\right), \nabla \psi\right) \tau \\
& -T_{0}\left(\mathbb{B} \partial_{t} \mathbf{u}_{n}(t), \nabla \psi\right)=\left(\bar{h}_{n}(t), \psi\right) .
\end{aligned}
$$

The Rellich-Kondrachov theorem [23, Theorem 1, p. 272] implies that $\mathrm{H}_{0}^{1}(\Omega) \hookrightarrow \hookrightarrow \mathrm{L}^{2}(\Omega) \hookrightarrow \hookrightarrow \mathrm{H}_{0}^{1}(\Omega)^{*}$. From (25), it follows that

$$
\max _{t \in[0, T]}\left\{\left\|\overline{\mathbf{u}}_{n}(t)\right\|_{\mathbf{H}_{0}^{1}(\Omega)}^{2}+\left\|\partial_{t} \mathbf{u}_{n}(t)\right\|^{2}\right\} \leqslant C .
$$

The conditions of [22, Lemma 1.3.13] are satisfied and, therefore, there exist a function $\mathbf{u} \in \mathrm{C}\left([0, T], \mathbf{L}^{2}(\Omega)\right) \cap$ $\mathrm{L}^{\infty}\left((0, T), \mathbf{H}_{0}^{1}(\Omega)\right)$ and a subsequence $\left\{\mathbf{u}_{n_{k}}\right\}_{k \in \mathbb{N}}$ of $\left\{\mathbf{u}_{n}\right\}_{n \in \mathbb{N}}$ (denoted by the same symbol yet again) such that

$$
\begin{cases}\mathbf{u}_{n} \rightarrow \mathbf{u} & \text { in } \mathrm{C}\left([0, T], \mathbf{L}^{2}(\Omega)\right), \\ \mathbf{u}_{n}(t) \rightarrow \mathbf{u}(t) & \text { in } \mathbf{H}_{0}^{1}(\Omega), \text { for all } t \in[0, T], \\ \overline{\mathbf{u}}_{n}(t) \rightarrow \mathbf{u}(t) & \text { in } \mathbf{H}_{0}^{1}(\Omega), \text { for all } t \in[0, T], \\ \partial_{t} \mathbf{u}_{n} \rightarrow \partial_{t} \mathbf{u} & \text { in } \mathrm{L}^{2}\left((0, T), \mathbf{L}^{2}(\Omega)\right) .\end{cases}
$$

Moreover, estimate (26) implies that

$$
\partial_{t} \mathbf{v}_{n} \rightarrow \partial_{t t} \mathbf{u} \quad \text { in } \mathrm{L}^{2}\left((0, T), \mathbf{H}_{0}^{1}(\Omega)^{*}\right)
$$

by the reflexivity of this space. The a priori estimate 25 implies that

$$
\int_{0}^{T}\left\|\mathbf{u}_{n}(t)-\overline{\mathbf{u}}_{n}(t)\right\|_{\mathbf{H}_{0}^{1}(\Omega)}^{2} \mathrm{~d} t=\sum_{i=1}^{n} \int_{t_{i-1}}^{t_{i}}\left\|\left(t-t_{i}\right) \delta \mathbf{u}_{i}\right\|_{\mathbf{H}_{0}^{1}(\Omega)}^{2} \mathrm{~d} t \leqslant \tau \sum_{i=1}^{n}\left\|\mathbf{u}_{i}-\mathbf{u}_{i-1}\right\|_{\mathbf{H}_{0}^{1}(\Omega)}^{2} \leqslant C \tau,
$$

i.e. $\left\{\mathbf{u}_{n}\right\}$ and $\left\{\overline{\mathbf{u}}_{n}\right\}$ have the same limit in $\mathrm{L}^{2}\left((0, T), \mathbf{H}_{0}^{1}(\Omega)\right)$.

From (25) and (26), it follows that

$$
\int_{0}^{T}\left\|\theta_{n}(t)\right\|_{\mathrm{H}_{0}^{1}(\Omega)}^{2} \mathrm{~d} t+\int_{0}^{T}\left\|\partial_{t} \theta_{n}(t)\right\|_{\mathrm{H}_{0}^{1}(\Omega)^{*}}^{2} \mathrm{~d} t \leqslant C .
$$

The generalized Aubin-Lions lemma [24, Lemma 2.12.4] implies the existence of a function $\theta \in \mathrm{L}^{2}\left((0, T), \mathrm{L}^{2}(\Omega)\right)$ and a subsequence $\left\{\theta_{n_{k}}\right\}_{n \in \mathbb{N}}$ of $\left\{\theta_{n}\right\}_{n \in \mathbb{N}}$ (denoted by the same symbol yet again) such that

$$
\begin{cases}\theta_{n} \rightarrow \theta & \text { in } \mathrm{L}^{2}\left((0, T), \mathrm{L}^{2}(\Omega)\right) \\ \theta_{n} \rightarrow \theta & \text { in } \mathrm{L}^{2}\left((0, T), \mathrm{H}^{1}(\Omega)\right) \\ \partial_{t} \theta_{n} \rightarrow \partial_{t} \theta & \text { in } \mathrm{L}^{2}\left((0, T), \mathrm{H}_{0}^{1}(\Omega)^{*}\right)\end{cases}
$$

Employing [25, Lemma 7.3] gives that $\theta \in \mathrm{C}\left([0, T], \mathrm{L}^{2}(\Omega)\right)$. Applying the a priori estimate [25], one obtains

$$
\int_{0}^{T}\left\|\theta_{n}(t)-\bar{\theta}_{n}(t)\right\|^{2} \mathrm{~d} t=\sum_{i=1}^{n} \int_{t_{i-1}}^{t_{i}}\left\|\left(t-t_{i}\right) \delta \theta_{i}\right\|^{2} \mathrm{~d} t \leqslant \tau \sum_{i=1}^{n}\left\|\theta_{i}-\theta_{i-1}\right\|^{2} \leqslant C \tau
$$


i.e. $\left\{\theta_{n}\right\}$ and $\left\{\bar{\theta}_{n}\right\}$ have the same limit in $\mathrm{L}^{2}\left((0, T), \mathrm{L}^{2}(\Omega)\right)$. Therefore, the a priori estimate 25 and the reflexivity of the space $\mathrm{L}^{2}\left((0, T), \mathrm{H}_{0}^{1}(\Omega)\right)$ imply that

$$
\bar{\theta}_{n} \rightarrow \theta \text { in } \mathrm{L}^{2}\left((0, T), \mathrm{H}_{0}^{1}(\Omega)\right) .
$$

From the reflexivity of the spaces $\mathrm{L}^{2}\left((0, T), \mathbf{L}^{2}(\Omega)\right)$ and $\mathrm{L}^{2}\left((0, T), \mathrm{L}^{2}(\Omega)\right)$, it follows that (in the sense of a subsequence being indexed by $n$ as well)

$$
\overline{\mathbf{r}}_{n} \rightarrow \mathbf{r} \text { in } \mathrm{L}^{2}\left((0, T), \mathbf{L}^{2}(\Omega)\right) \quad \text { and } \quad \bar{h}_{n} \rightarrow h \text { in } \mathrm{L}^{2}\left((0, T), \mathrm{L}^{2}(\Omega)\right) .
$$

Finally, the existence of a solution can be proven. In doing this, we integrate (27) and (28) in time and pass to the limit for $\tau \rightarrow 0$ using the preceding convergence results. Afterwards, we differentiate the result with respect to the time variable to arrive at (18)-(19). The convergence of Rothe's functions towards the weak solution has been shown for a subsequence. However, taking into account the uniqueness of a solution, the entire Rothe's sequence converges towards the solution.

The estimate (20) can be derived as follows. Firstly, we choose $\varphi=\partial_{t} \mathbf{u}(t)$ and $\psi=\frac{\theta(t)}{T_{0}}$ in (18)-(19) and integrate in time over $t \in(0, \eta) \subset(0, T)$. We then sum up the resulting equations to obtain

$$
\begin{aligned}
& \frac{\left\|\sqrt{\rho} \partial_{t} \mathbf{u}(\eta)\right\|^{2}}{2}+\int_{0}^{\eta}\left\|\sqrt{g} \partial_{t} \mathbf{u}(t)\right\|^{2} \mathrm{~d} t+\frac{1}{2}\left(\mathcal{L}^{\mathrm{e}} \mathbf{u}(\eta), \mathbf{u}(\eta)\right)+\frac{\left\|\sqrt{\varrho C_{s}} \theta(\eta)\right\|^{2}}{2 T_{0}} \\
& +\frac{1}{T_{0}} \int_{0}^{\eta}(\mathbb{K} \nabla \theta(t), \nabla \theta(t)) \mathrm{d} t+\frac{1}{T_{0}} \int_{0}^{\eta}((K * \nabla \theta)(t), \nabla \theta(t)) \mathrm{d} t=\int_{0}^{\eta}\left(\mathbf{r}(t), \partial_{t} \mathbf{u}(t)\right) \mathrm{d} t \\
& +\frac{\left\|\sqrt{\rho} \overline{\mathbf{u}}_{1}\right\|^{2}}{2}+\frac{1}{2}\left(\mathcal{L}^{\mathrm{e}} \overline{\mathbf{u}}_{0}, \overline{\mathbf{u}}_{0}\right)+\frac{1}{T_{0}} \int_{0}^{\eta}(h(t), \theta(t)) \mathrm{d} t+\frac{\left\|\sqrt{\varrho C_{s}} \bar{\theta}_{0}\right\|^{2}}{2} .
\end{aligned}
$$

Similarly to the a priori estimates $(25)$, one can show that

$$
\max _{t \in[0, T]}\left\{\left\|\partial_{t} \mathbf{u}(t)\right\|^{2}+\|\mathbf{u}(t)\|_{\mathbf{H}_{0}^{1}(\Omega)}^{2}+\|\theta(t)\|^{2}\right\}+\int_{0}^{T}\|\nabla \theta(t)\|^{2} \mathrm{~d} t \leqslant C,
$$

where $C=C\left(\left\|\overline{\mathbf{u}}_{0}\right\|_{\mathbf{H}_{0}^{1}(\Omega)},\left\|\overline{\mathbf{u}}_{1}\right\|,\left\|\bar{\theta}_{0}\right\|_{\mathrm{H}^{1}(\Omega)},\|\mathbf{r}\|_{\mathrm{L}^{2}\left((0, T), \mathbf{L}^{2}(\Omega)\right)},\|h\|_{\mathrm{L}^{2}\left((0, T), \mathrm{L}^{2}(\Omega)\right)}\right)$. From this estimate, it follows 20, provided that $\overline{\mathbf{u}}_{0}(\mathbf{x})=\mathbf{0}, \overline{\mathbf{u}}_{1}(\mathbf{x})=\mathbf{0}, \bar{\theta}_{0}=0, h=0$ and $\mathbf{r}=\mathbf{r}(\mathbf{x})$.

(ii) and (iii) The a priori estimates (25) and (26) also remain valid under the assumption (ii) or (iii), the only difference being the estimation of term containing values of $K$. In the first case, on using the Young's inequality for discrete convolutions, one obtains

$$
\sum_{i=1}^{j}\left(\sum_{l=1}^{i} K_{l} \nabla \theta_{i-l} \tau, \nabla \theta_{i}\right) \tau \leqslant\left(\sum_{i=1}^{j}\left|K_{i}\right| \tau\right) \sum_{i=1}^{j}\left\|\nabla \theta_{i}\right\|^{2} \tau,
$$

whilst in the second case, it follows that

$$
\sum_{i=1}^{j}\left(\sum_{l=1}^{i} K_{l} \nabla \theta_{i-l} \tau, \nabla \theta_{i}\right) \tau \leqslant C_{\varepsilon} \sum_{i=1}^{j}\left(\sum_{l=1}^{i}\left\|\nabla \theta_{i}\right\|^{2}\right) \tau+\varepsilon \sum_{i=1}^{j}\left\|\nabla \theta_{i}\right\|^{2} \tau .
$$

It should be mentioned that the application of Grönwall's lemma is required to obtain 25 in the latter case. 
In the following, it is assumed that the hypothesis (i), (ii) or (iii) of Theorem 4.1 is valid. This implies, in particular, that the boundary conditions are satisfied and the final displacement measurement is well-defined (as an element of $\mathrm{L}^{2}(\Omega)$ ). In the following subsection, the algorithm for the recovery of the unknown source term is proposed. Without any loss of the generality, it is assumed that the following problem is studied:

$$
\begin{cases}\varrho(\mathbf{x}) \partial_{t t} \mathbf{u}(\mathbf{x}, t)+g(\mathbf{x}) \partial_{t} \mathbf{u}(\mathbf{x}, t)-\mathcal{L}^{\mathrm{e}} \mathbf{u}(\mathbf{x}, t)+\operatorname{div}(\mathbb{B}(\mathbf{x}) \theta(\mathbf{x}, t))=\mathbf{p}(\mathbf{x}), & (\mathbf{x}, t) \in Q_{T}, \\ \varrho(\mathbf{x}) C_{s}(\mathbf{x}) \partial_{t} \theta(\mathbf{x}, t)-\nabla \cdot(\mathbb{K}(\mathbf{x}) \nabla \theta(\mathbf{x}, t))-(K * \Delta \theta)(\mathbf{x}, t)+T_{0} \mathbb{B}(\mathbf{x}): \nabla \partial_{t} \mathbf{u}(\mathbf{x}, t)=0, & (\mathbf{x}, t) \in Q_{T}, \\ \mathbf{u}(\mathbf{x}, t)=\mathbf{0}, & (\mathbf{x}, t) \in \Sigma_{T}, \\ \theta(\mathbf{x}, t)=0, & (\mathbf{x}, t) \in \Sigma_{T}, \\ \mathbf{u}(\mathbf{x}, 0)=\partial_{t} \mathbf{u}(\mathbf{x}, 0)=\mathbf{0}, \quad \theta(\mathbf{x}, 0)=0, & \mathbf{x} \in \Omega, \\ \mathbf{u}(\mathbf{x}, T)=\boldsymbol{\xi}_{T}(\mathbf{x}), & \mathbf{x} \in \Omega,\end{cases}
$$

where the final time measurement has been denoted by the same symbol yet again.

\subsection{Algorithm for finding the source term}

In this section, an algorithm for finding the source term is described. This algorithm is based on an iterative regularization method, namely the Landweber-Fridman iteration [26, 27].

Let $(\mathbf{u}, \theta)$ the unique solution to (30) for given $\mathbf{p}$, see Theorem 4.1. Define the corresponding operator $M(t) \in \mathcal{L}\left(\mathbf{L}^{2}(\Omega), \mathbf{L}^{2}(\Omega)\right)$ by

$$
M(t) \mathbf{p}=\mathbf{u}(\cdot, t) .
$$

Finding a solution to the inverse problem is then equivalent to solving the following operator equation

$$
M(T) \mathbf{p}=\boldsymbol{\xi}_{T},
$$

or solving the following fixed point equation

$$
\mathbf{p}=\mathbf{p}+\omega M(T)\left(\boldsymbol{\xi}_{T}-M(T) \mathbf{p}\right), \quad \omega>0,
$$

due to the linearity of the operator $M(T)$. The parameter $\omega$ is called a relaxation parameter. The method of successive approximations can be applied to this latter equation as follows

$$
\mathbf{p}_{k}:=\mathbf{p}_{k-1}-\omega M(T)\left(M(T) \mathbf{p}_{k-1}-\boldsymbol{\xi}_{T}\right), \quad k \in \mathbb{N},
$$

with an initial guess $\mathbf{p}_{0}$.

This gives rise to the following procedure for the stable reconstruction of the solution $(\mathbf{u}, \theta)$ and the source term $\mathbf{p}$ of problem (1)-(3). This procedure is similar to that presented in [14, 28-30] and reads as follows:

(i) Choose an initial guess $\mathbf{p}_{0} \in \mathbf{L}^{2}(\Omega)$. Let $\left(\mathbf{u}_{0}, \theta_{0}\right)$ be the solution to $(30)$ with $\mathbf{p}=\mathbf{p}_{0}$.

(ii) Assume that $\mathbf{p}_{k-1}$ and $\left(\mathbf{u}_{k-1}, \theta_{k-1}\right)$ have been constructed. Let $\left(\mathbf{v}_{k-1}, \eta_{k-1}\right)$ solve 30 with $\mathbf{p}(\mathbf{x})=$ $\mathbf{u}_{k-1}(\mathbf{x}, T)-\boldsymbol{\xi}_{T}(\mathbf{x})$.

(iii) Define

$$
\mathbf{p}_{k}(\mathbf{x})=\mathbf{p}_{k-1}(\mathbf{x})-\omega \mathbf{v}_{k-1}(\mathbf{x}, T), \quad \mathbf{x} \in \Omega
$$

where $\omega>0$, and let $\left(\mathbf{u}_{k}, \theta_{k}\right)$ solve (30) with $\mathbf{p}=\mathbf{p}_{k}$.

(iv) Repeat steps (ii) and (iii) until a desired level of accuracy is achieved, see Subsections 4.2 and 4.3 .

The problems used in this iterative procedure are well-posed, see Theorem 4.1. Moreover, the restrictions of solutions are well-defined. The convergence of the procedure is studied in the following subsection. 


\subsection{Convergence of the proposed algorithm in Subsection 4.1}

The following theorem shows the convergence of the proposed algorithm in the previous subsection.

Theorem 4.2 (Existence). Assume that the assumptions of Theorem 4.1 are satisfied and suppose that the relaxation parameter $\omega$ satisfies $0<\omega<\|M(T)\|^{-2}$. Denote by $(\mathbf{u}, \theta, \mathbf{p})$ the unique solution to the original inverse problem (1)-(3). Let $\left(\mathbf{u}_{k}, \theta_{k}, \mathbf{p}_{k}\right)$ the $k$-th approximation in the iterative algorithm of Subsection 4.1 Then

$$
\lim _{k \rightarrow \infty}\left\{\left\|\mathbf{u}-\mathbf{u}_{k}\right\|_{\mathrm{C}\left([0, T], \mathbf{L}^{2}(\Omega)\right)}+\left\|\theta-\theta_{k}\right\|_{\mathrm{C}\left([0, T], \mathrm{L}^{2}(\Omega)\right)}\right\}=0
$$

for every function $\mathbf{p}_{0} \in \mathbf{L}^{2}(\Omega)$.

Proof. From the iterative algorithm and the linearity of the operator $M(t)$, it follows that

$$
\begin{aligned}
\mathbf{p}_{k} & =\mathbf{p}_{k-1}-\omega \mathbf{v}_{k-1}(\cdot, T)=\mathbf{p}_{k-1}-\omega M(T)\left(\mathbf{u}_{k-1}(\cdot, T)-\boldsymbol{\xi}_{T}\right) \\
\stackrel{31]}{=} & \mathbf{p}_{k-1}-\omega M(T)\left(M(T) \mathbf{p}_{k-1}-M(T) \mathbf{p}\right)=\mathbf{p}_{k-1}-\omega M(T) M(T)\left(\mathbf{p}_{k-1}-\mathbf{p}\right) .
\end{aligned}
$$

Therefore,

$$
\mathbf{p}_{k}-\mathbf{p}=(I-\omega M(T) M(T))\left(\mathbf{p}_{k-1}-\mathbf{p}\right) .
$$

This is the Landweber-Fridman iterative scheme for solving the operator equation (31). The proof of convergence for the Landweber-Fridman iteration in the case of two not self-adjoint operators is given in [31, Theorem 3]. Since $0<\omega<\|M(T)\|^{-2}$, it follows that $\mathbf{p}_{k}$ converges to $\mathbf{p}$ in $\mathbf{L}^{2}(\Omega)$, for an arbitrary $\mathbf{p}_{0} \in \mathbf{L}^{2}(\Omega)$. Inequality (20) implies the convergence of $\mathbf{u}_{k}$ and $\theta_{k}$ to the corresponding solution in the given spaces.

\subsection{Stopping criterion}

Reconsider the algorithm presented in Subsection 4.1. Herein, the displacement at the final time is measured to obtain a solution to problem (1)-(2). To model each practical experiment in a realistic manner, it is assumed that there exists some level of error in the additional measurement (3), i.e.

$$
\left\|\boldsymbol{\xi}_{T}-\boldsymbol{\xi}_{T}^{e}\right\| \leqslant e
$$

with $e>0$. Note that functions $\mathbf{p}_{k}^{e}, \mathbf{u}_{k}^{e}$ and $\theta_{k}^{e}$ are obtained by using the algorithm without noise in the initial data (2). The absolute $\mathbf{L}^{2}$-error between this final measurement $\boldsymbol{\xi}_{T}^{e}$ and the $k$-th approximation $\mathbf{u}_{k}^{e}(\cdot, t)$ at $t=T$ is denoted by

$$
E_{k, \mathbf{u}_{T}}=\left\|\mathbf{u}_{k}^{e}(\cdot, T)-\boldsymbol{\xi}_{T}^{e}\right\| .
$$

Given the noise level $e$, Morozov's discrepancy principle [32] is used to obtain a stopping criterion for the algorithm. This principle suggests to stop the iterations at the lowest index $k=k(e, \omega)$ for which

$$
E_{k, \mathbf{u}_{T}} \leqslant e .
$$




\section{Numerical experiments}

In the following, we consider an isotropic homogeneous thermoelastic material that occupies an open and bounded domain $\Omega \subset \mathbb{R}^{d}$, with $d=1$ (Experiments 1 and 2) and $d=2$ (Experiment 3). For such a material, the elasticity tensor is given by

$$
C_{i j k l}:=\lambda \delta_{i j} \delta_{k l}+\mu \delta_{i k} \delta_{j l}+\mu \delta_{i l} \delta_{j k}, \quad 1 \leq i, j, k, l \leq d,
$$

where $\lambda>0$ (SI unit: $\mathrm{N} / \mathrm{m}^{2}$ ) and $\mu>0$ (SI unit: $\mathrm{N} / \mathrm{m}^{2}$ ) are the so-called Lamé parameters. These quantities can be expressed in terms of the shear modulus $G>0$ and Poisson's ratio $\nu \in(0,0.5)$ as

$$
\lambda=\frac{2 \nu G}{1-2 \nu} \quad \text { and } \quad \mu=G .
$$

In this case, the thermal conductivity $\mathbb{K}(\mathrm{SI}$ unit: $\mathrm{W} / \mathrm{mK})$ and thermoelasticity $\mathbb{B}$ tensors are given by

$$
K_{i j}=\kappa \delta_{i j}, \quad \text { and } \quad b_{i j}(x)=\beta \delta_{i j}, \quad 1 \leq i, j \leq d,
$$

respectively. Here, $\kappa>0$ and the coupling parameter $\beta>0\left(\right.$ SI unit: $\left.\mathrm{N} / \mathrm{m}^{2} \mathrm{~K}\right)$ is defined as

$$
\beta=\alpha_{T}(3 \lambda+2 \mu)=2 \alpha_{T} G \frac{1+\nu}{1-2 \nu},
$$

where $\alpha_{T}>0$ (SI unit: $1 / \mathrm{K}$ ) is the coefficient of linear expansion of the thermoelastic material.

Consequently, problem (1) becomes

$$
\begin{cases}\varrho \partial_{t t} \mathbf{u}(\mathbf{x}, t)+g \partial_{t} \mathbf{u}(\mathbf{x}, t)-\mu \Delta \mathbf{u}(\mathbf{x}, t)-(\mu+\lambda) \nabla(\nabla \cdot \mathbf{u}(\mathbf{x}, t))+\beta \nabla \theta(\mathbf{x}, t)=\mathbf{r}(\mathbf{x}, t)+\mathbf{p}(\mathbf{x}), & (\mathbf{x}, t) \in Q_{T}, \\ \varrho C_{s} \partial_{t} \theta(\mathbf{x}, t)-\kappa \Delta \theta(\mathbf{x}, t)-(K * \Delta \theta)(\mathbf{x}, t)+T_{0} \beta \nabla \cdot \partial_{t} \mathbf{u}(\mathbf{x}, t)=h(\mathbf{x}, t), & (\mathbf{x}, t) \in Q_{T} .\end{cases}
$$

The material constants employed in the numerical experiments have been taken so that they correspond to a copper alloy [33], namely $G=4.8 \times 10^{10} \mathrm{~N} / \mathrm{m}^{2}, \nu=0.34, \alpha_{T}=16.5 \times 10^{-6} 1 / \mathrm{K}, \kappa=401 \mathrm{~W} / \mathrm{mK}$, $\rho=8960 \mathrm{~kg} / \mathrm{m}^{3}$ and $C_{s}=385 \mathrm{~J} / \mathrm{kgK}$. In order to preserve the coercivity, the damping coefficient $g$ (SI unit: $\mathrm{kg} / \mathrm{m}^{3} \mathrm{~s}$ ) has been taken to be $g=2 \times 10^{8}$ in $1 \mathrm{D}$ and $g=2 \times 10^{14}$ in $2 \mathrm{D}$, respectively, whilst in all experiments performed herein, $T_{0}=293 \mathrm{~K}$.

It should be mentioned that the governing equations in (34) can be rearranged in a more convenient form by using the following nondimensional variables:

$$
\mathbf{x}^{\prime}=\frac{1}{\ell} \mathbf{x}, \quad t^{\prime}=\frac{C_{1}}{\ell} t, \quad \theta^{\prime}\left(\mathbf{x}^{\prime}, t^{\prime}\right)=\frac{1}{T_{0}} \theta(\mathbf{x}, t), \quad \mathbf{u}^{\prime}\left(\mathbf{x}^{\prime}, t^{\prime}\right)=\frac{\lambda+2 \mu}{\beta T_{0} \ell} \mathbf{u}(\mathbf{x}, t)
$$

where $C_{1}=\sqrt{(\lambda+2 \mu) / \rho}$ (SI unit: $\mathrm{m} / \mathrm{s}$ ) and $\ell=\kappa /\left(\rho C_{s} C_{1}\right)$ (SI unit: $\mathrm{m}$ ). The dimensionless variables are used in the following experiments, however, in the sequel, the accent is dropped in order to simplify the notations employed.

The solution to the inverse source problems is found by applying the algorithm proposed in Subsection 4.1. As described in the proof of Theorem 4.1, the forward mixed problems in this procedure are discretized in time according to the backward Euler method. It is assumed that $T=1$ in $1 \mathrm{D}$ and $T=0.5$ in $2 \mathrm{D}$. The time step for the equidistant time partitioning is chosen to be 0.0005 in $1 \mathrm{D}$ and 0.0001 in 2D. At each time step, the resulting elliptic mixed problems, see (21) and (22), are solved numerically by the finite element method using first order (P1-FEM) Lagrange polynomials for the space discretization (the number of 1D 
and 2D finite elements was taken to be equal to 200 and 5000, respectively). For these calculations, the finite element library DOLFIN [34, 35] from the FEniCS project [36, 37] is used.

For almost all examples considered, a randomly generated uncorrelated noise is added to the additional condition in order to simulate the inherent errors present in real measurements. The resulting final measurement is denoted by $\boldsymbol{\xi}_{T}^{e}$, see also Subsection 4.3. In the following, the exact value for the source $\mathbf{p}$ is compared its corresponding numerically retrieved value $\mathbf{p}_{\tilde{k}}$, obtained when the algorithm is stopped after a finite number of iterations $\tilde{k}$. The initial guess $\mathbf{p}_{0}$ is chosen to be equal to $\mathbf{0}$ unless otherwise specified. The algorithm has the following stopping criterion:

$$
E_{k, \mathbf{u}_{T}} \leqslant e, \quad \frac{\left|E_{k, \mathbf{u}_{T}}-E_{k-1, \mathbf{u}_{T}}\right|}{E_{k, \mathbf{u}_{T}}} \leqslant 10^{-4},
$$

and maximum 10000 iterations.

\subsection{Experiment 1: $1 D$ problem in a $2 D$ setting with a smooth and symmetric variation of the load vector}

The 1D linear model for an isotropic thermoelastic material in the domain $\Omega=(0,1)$ is given by: Find $(u, \theta, p)$ such that

$$
\begin{cases}u_{t t}(x, t)+g_{2} u_{t}(x, t)-u_{x x}(x, t)+\theta_{x}(x, t)=r(x, t)+p(x) & (x, t) \in Q_{T}, \\ \theta_{t}(x, t)-\theta_{x x}(x, t)-k_{2}\left(K * \theta_{x x}\right)(x, t)+\varepsilon_{2} u_{x t}(x, t)=h(x, t) & (x, t) \in Q_{T}, \\ u(0, t)=u(1, t)=0 & t \in(0,1], \\ \theta(0, t)=\theta(1, t)=0 & t \in(0,1], \\ u(x, 0)=\bar{u}_{0}(x), \quad u_{t}(x, 0)=\bar{u}_{1}(x), & x \in(0,1), \\ \theta(x, 0)=\bar{\theta}_{0}(x), & x \in(0,1),\end{cases}
$$

and the final over-determination condition is satisfied

$$
u(x, T)=\xi_{T}^{e}(x), \quad x \in(0,1),
$$

with

$$
g_{2}:=\frac{g}{\sqrt{\varrho(\lambda+2 \mu)}}, \quad k_{2}:=\frac{1}{\rho C_{s} C_{1}^{2}}, \quad \varepsilon_{2}:=\frac{\beta^{2} T_{0}}{\varrho^{2} C_{s} C_{1}^{2}} .
$$

The exact solution to problem (35)-(36) is prescribed as follows

$$
\begin{aligned}
& u(x, t)=(1+t)^{2} x(x-1)^{2}, \quad \theta(x, t)=(1+t) x(1-x)^{2}, \\
& p_{1}(x)=10 x(x-1) \quad \text { or } \quad p_{2}(x)=\exp \left(-20(x-0.5)^{2}\right) .
\end{aligned}
$$

Thus two experiments, depending on the unknown space source $p_{1}$ or $p_{2}$, are considered. Moreover, three choices for the convolution kernel are made, namely $K=0, K=\exp (-t)$ and $K=1 / \sqrt{t}$, respectively. This distinction is based on the theoretical analysis. The error $e$ on the final measurement is given in Table 1 (for different noise levels with magnitude $\tilde{e}$ ). The experiments are performed with relaxation parameter $\omega=10$. The stopping iteration index and the CPU time (in minutes) for various experiments can be found in Table 2. The numerical results for different noise levels $\tilde{e}$ are depicted in Figure 1 when $p_{1}$ is unknown and in Figure 2 when $p_{2}$ is unknown. The obtained results are in accordance with the numerical experiments performed for the heat conduction equation in [28, 30] and for type-I thermoelasticity $(K=0)$ in [14]. The attainability of the stopping criterion becomes faster if $\tilde{e}$ increases. For each noise level and the different 
Table 1: The error $e(\tilde{e}) \approx\left\|\xi_{T}-\xi_{T}^{e}\right\|$, obtained for Experiment 1, with the unknown sources $p_{1}$ and $p_{2}$.

\begin{tabular}{c|ccc|ccc}
\hline & \multicolumn{3}{|c|}{$p_{1}$} & \multicolumn{3}{c}{$p_{2}$} \\
Kernel \e & $1 \%$ & $5 \%$ & $10 \%$ & $0.5 \%$ & $1 \%$ & $3 \%$ \\
\hline$K=0$ & 0.004928 & 0.023689 & 0.042853 & 0.002490 & 0.004554 & 0.014829 \\
$K=\exp (-t)$ & 0.004580 & 0.023746 & 0.044725 & 0.002335 & 0.004602 & 0.013740 \\
$K=1 / \sqrt{t}$ & 0.004697 & 0.023135 & 0.044462 & 0.002395 & 0.004742 & 0.014753 \\
\hline
\end{tabular}

Table 2: The stopping iteration number $\tilde{k}=k(e(\tilde{e}), 10)$ and the CPU time (mins), obtained for Experiment 1 , with the unknown sources $p_{1}$ and $p_{2}$.

kernels, an accurate approximation for the source $p_{1}$ is obtained. In the case of $10 \%$ noise, the accurate approximation of the source shows the stability of the numerical procedure. The shape of the source $p_{2}$ can be recovered when the magnitude of the noise is around 3\%. Although not presented herein, it is reported that the algorithm is more sensitive to increasing the amount of noise for this experiment.

\subsection{Experiment 2: $1 D$ problem in a $2 D$ setting with a nonsmooth and/or a nonsymmetric variation of the load vector}

Herein, the exact solution to problem $(35)-(36)$ is given by

$$
u(x, t)=(1+t)^{2} x(x-1)^{2}, \quad \theta(x, t)=(1+t) x(1-x)^{2},
$$

with

$$
p_{3}(x)=\left\{\begin{array}{ll}
0 & 0 \leqslant x \leqslant \frac{1}{3} \\
6 x-2 & \frac{1}{3} \leqslant x \leqslant \frac{1}{2} \\
4-6 x & \frac{1}{2} \leqslant x \leqslant \frac{2}{3} \\
0 & \frac{2}{3} \leqslant x \leqslant 1
\end{array}, \quad p_{4}(x)= \begin{cases}x(0.5-x)(1-x) \quad 0 \leqslant x \leqslant \frac{1}{2} \\
x(x-0.5)(1-x) \quad \frac{1}{2} \leqslant x \leqslant 1\end{cases}\right.
$$

$$
p_{5}(x)= \begin{cases}0 & 0 \leqslant x<\frac{1}{3} \\ 1 & \frac{1}{3} \leqslant x \leqslant \frac{2}{3}, \quad p_{6}(x)=10 x(x-1)^{2} . \\ 0 & \frac{2}{3}<x \leqslant 1\end{cases}
$$

Sources $p_{3}$ and $p_{4}$ are continuous piecewise smooth, whilst $p_{5}$ is discontinuous. The first three sources are symmetric and this in contrast to the source $p_{6}$, which is non-symmetric (and smooth). We only show the outcome of the numerical experiments for $K=0$ because the results obtained for the experiments performed 


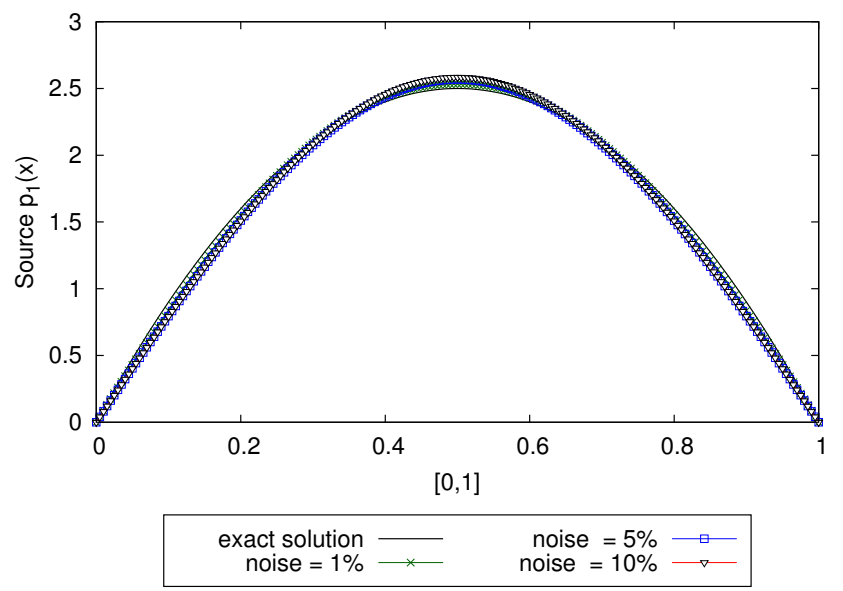

(a)

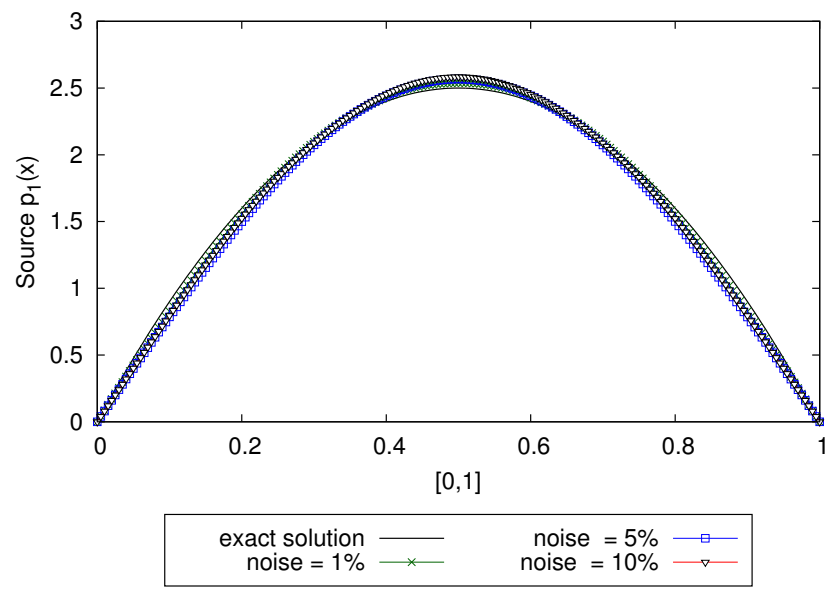

(b)

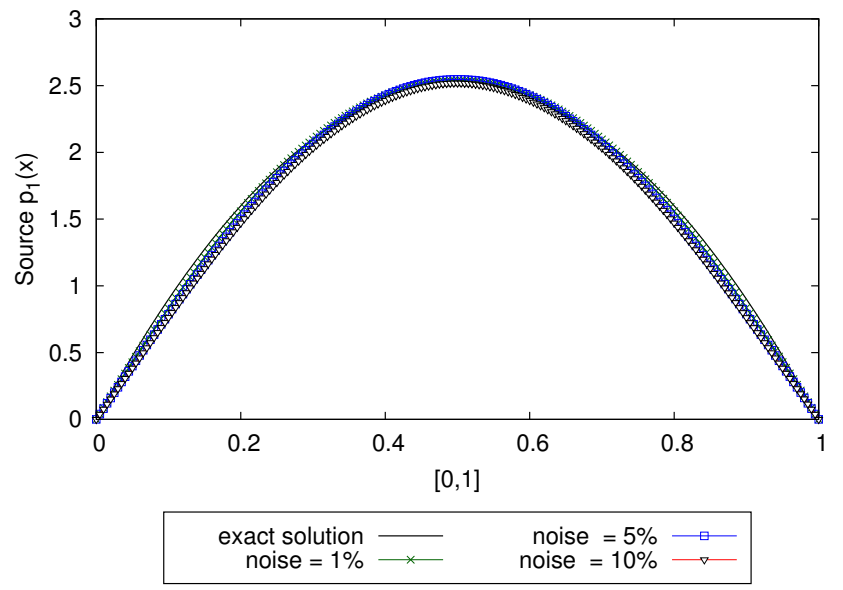

(c)

Figure 1: Example 1: The exact source $p_{1}$ and its corresponding numerical solution, retrieved using various levels of noise in the additional measurement (3), for various convolution kernels, namely (a) $K=0$, (b) $K=\exp (-t)$, and (c) $K=1 / \sqrt{t}$. 


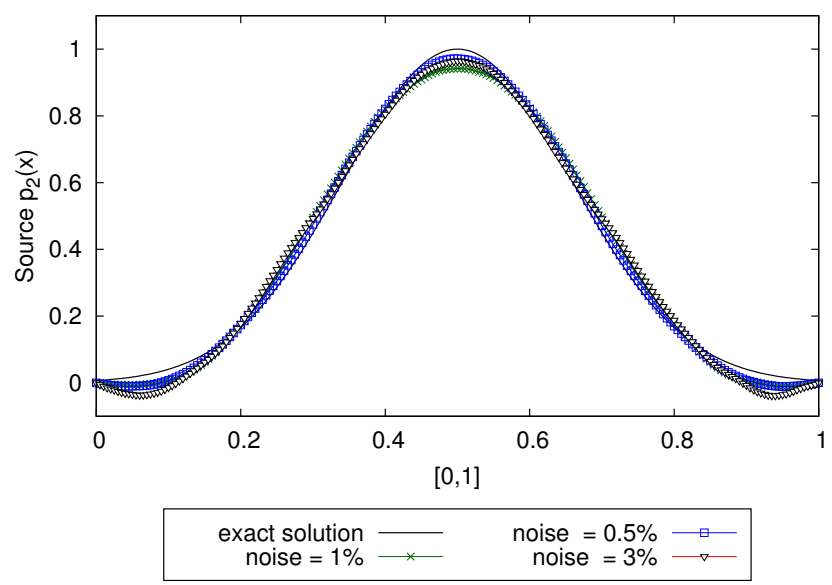

(a)

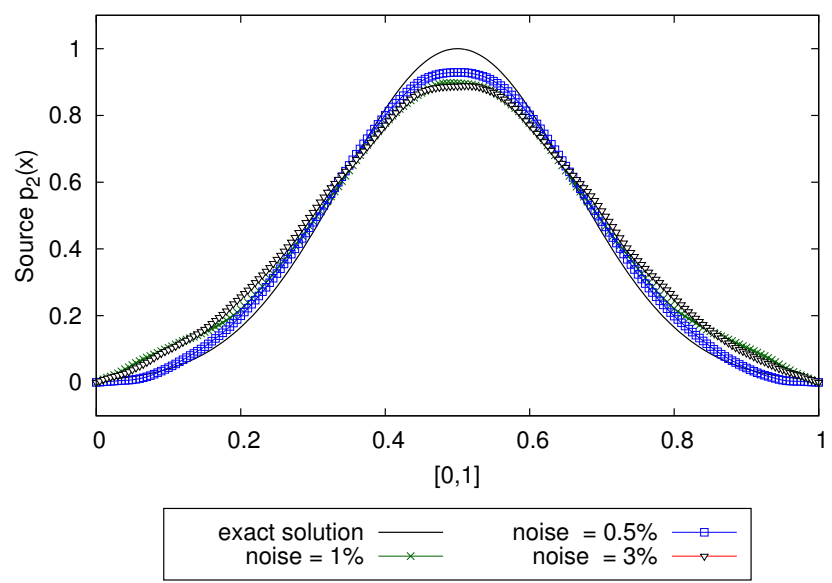

(b)

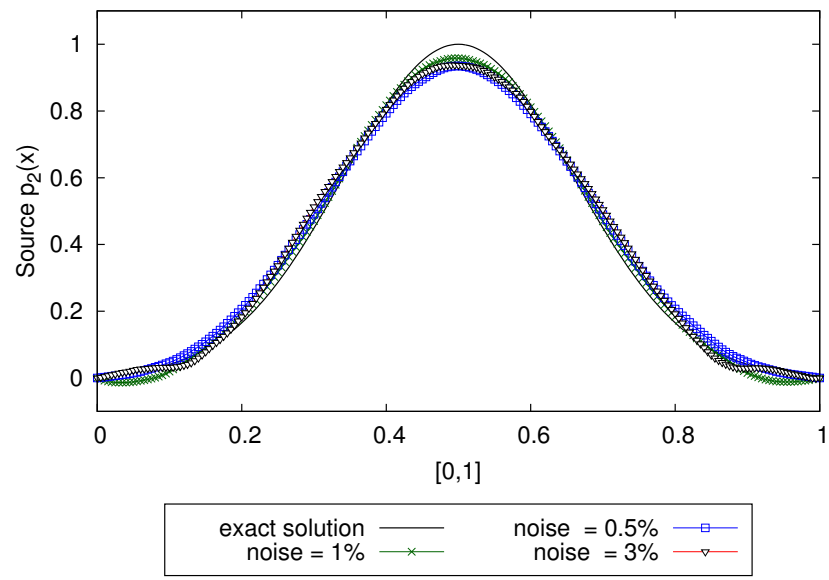

(c)

Figure 2: Example 1: The exact source $p_{2}$ and its corresponding numerical solution, retrieved using various levels of noise in the additional measurement (3), for various convolution kernels, namely (a) $K=0$, (b) $K=\exp (-t)$, and (c) $K=1 / \sqrt{t}$. 
with $K=\exp (-t)$ and $K=1 / \sqrt{t}$ are similar. The main difference is that the CPU time is larger for these latter choices of the kernel as already noticed from Table 2

First, we present the results for the experiments related to $p_{3}, p_{4}$ and $p_{5}$ when exact data is used, i.e. $\tilde{e}=$ $0 \%$. In this case, the iterative process is continued indefinitely. In Figures 3(a), (c) and (e), the numerical solution for these sources is compared to their corresponding exact values for various iteration numbers $k \in\{500,1000,2000,5000,10000\}$ and $\omega=10$. From these figures, it can be seen that the numerical solution converges to its corresponding exact solution as $k$ increases. However, this process is very slow as each numerical experiment requires about ten hours for 1000 iterations. In addition, in Figures 3 (b), (d) and (f), numerical approximations are presented for various noise levels and $\omega=10$. Again, reasonable numerical approximations are obtained. Furthermore, from Figure 3(d) it is clear that the algorithm is sensitive to the amount of noise added to the data. Note that for the experiment corresponding to source $p_{4}$, the cut-off stopping criteria is not taken into account in order to obtain better results. For the sake of completeness, for this experiment the results retrieved for $\omega=2$ and $\omega=20$ are also included, see Figure 4. The results for $\tilde{e}=0.1 \%$ are similar. Future research will be devoted to investigating the influence of the smoothing parameter $g$ on the reconstruction of the source. In Figure 5, source $p_{5}$ is reconstructed for various values of $g$. For this experiment the results seem to be analogous for the different values of $g$. Figure 6 suggests that choosing $g=2 \times 10^{8}$ seems to be an appropriate choice, however further investigation is required.

The numerical experiment corresponding to $p_{6}$ suggests a certain limitation of the method with respect to the recovery of non-symmetric sources. With zero initial guess and $\omega=10$, the numerical procedure gives an unsatisfactory (not usable) approximation, see Figure 7(a). Experiments with the same initial guess and a lower level of noise and/or a lower value of the relaxation parameter give similar results. Furthermore, we have also considered the following range of non-symmetric initial guesses: $6.44 x-12.27 x^{2}+5.83 x^{3}$, $9.68 x-18.46 x^{2}+8.78 x^{3}$ and $12.88 x-24.54 x^{2}+11.65 x^{3}$, obtained by a least-squares approximation through points $(0,0),(1,0)$ and $\left(\frac{1}{3}, 1\right),\left(\frac{1}{3}, 1.5\right),\left(\frac{1}{3}, 2\right)$, respectively. The results are depicted in Figures $7(\mathrm{~b}-$ d). From these figures it can be seen that, as expected, a better numerical approximation is obtained when the initial guess is closer to the exact solution. This example is somehow in contradiction with the algorithm, in the sense that there is no limitation on the initial guess in the algorithm. A possible explanation might be the fact that the timestep is not small enough for this type of source such that the errors in the numerical solution accumulates. However, experiments performed with a smaller timestep do not give better results and they are thus not confirming this reasoning. Instead of making the timestep smaller, in the following experiment the final time is reduced to $T=0.2$ and $T=0.5$ (hence the exact solution becomes linear in time). The results retrieved from this experiment are displayed in Figure 8 . For both situations, good numerical approximations are obtained when the noise level is small.

From the experiments performed in this subsection, we conclude that the proposed numerical algorithm is at least applicable to the reconstruction of symmetric load fields with discontinuities and symmetric continuous piecewise smooth load fields. For experiments with non-symmetric load fields, the experiments suggest that the final time should be small enough in order to obtain good results for our test example. The aforementioned limitation will be further investigated in the future. 


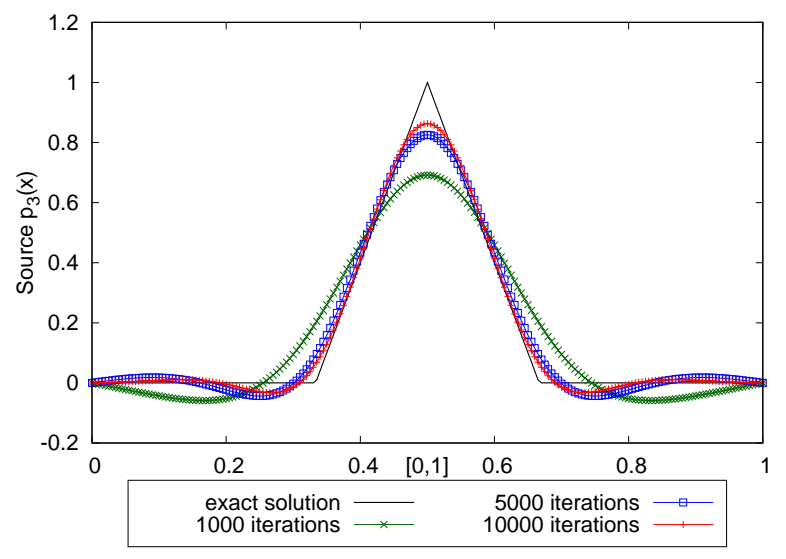

(a)

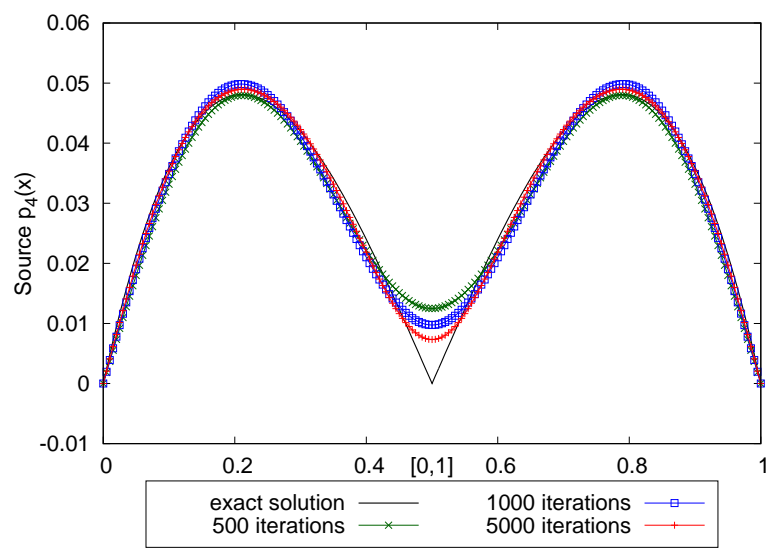

(c)

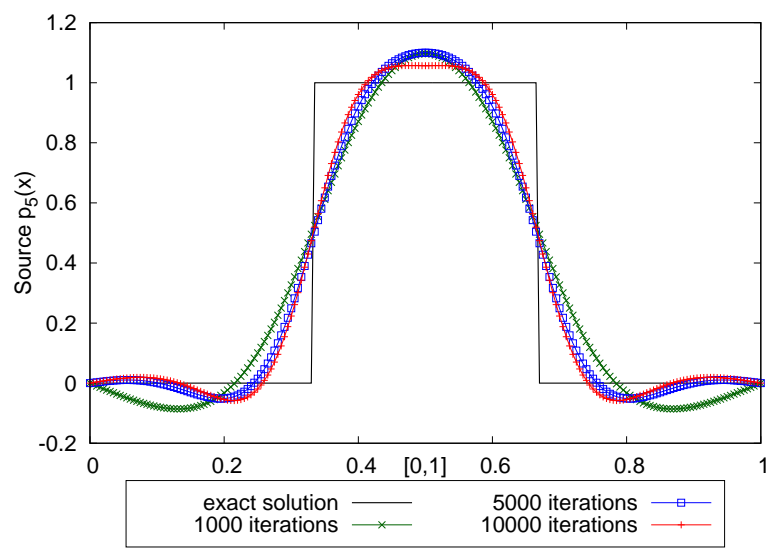

(e)

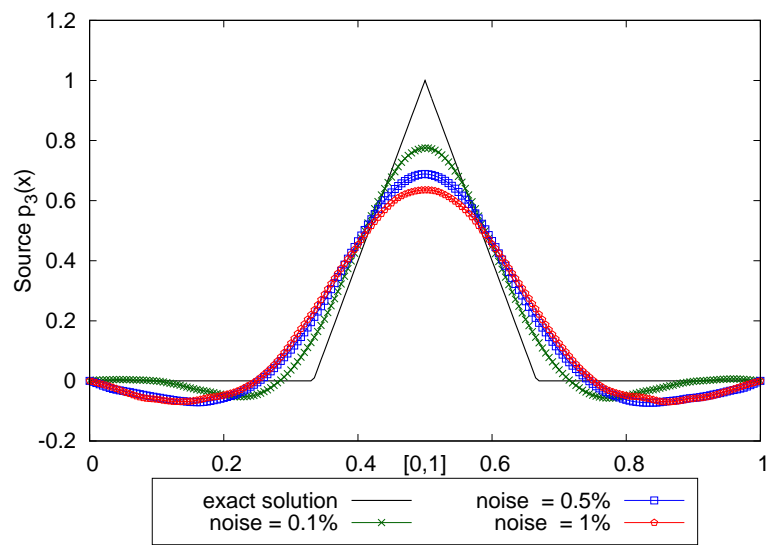

(b)

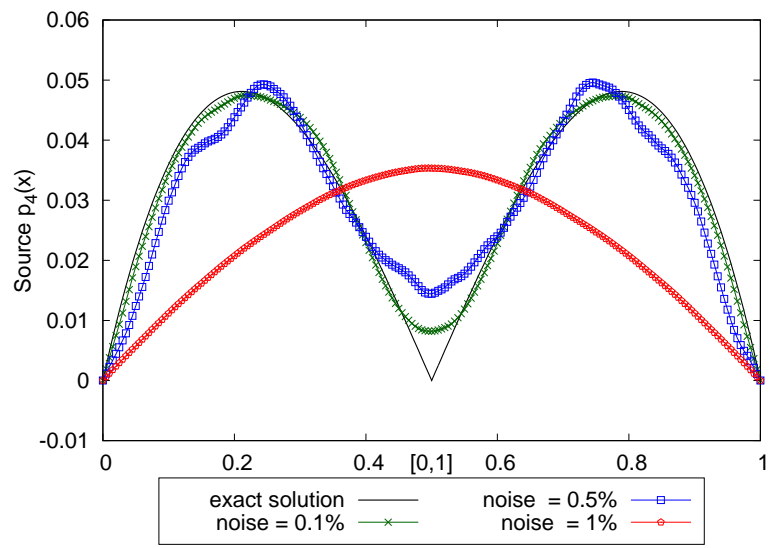

(d)

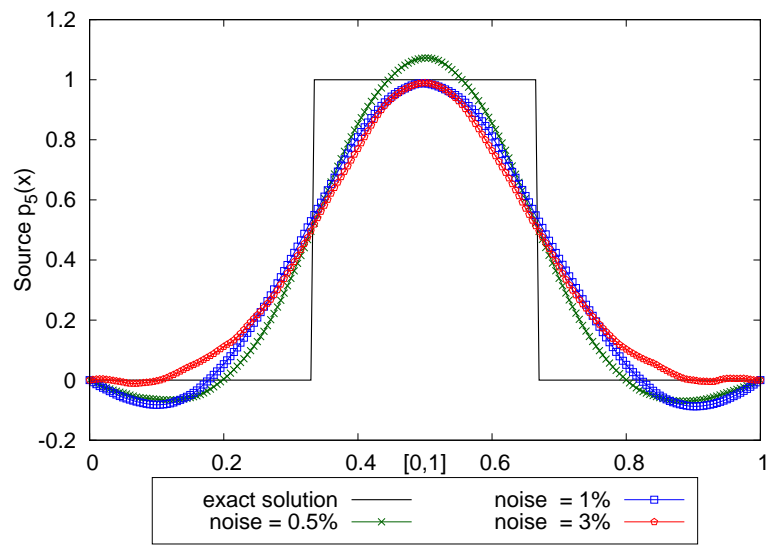

(f)

Figure 3: Example 2: The exact sources $p_{3}, p_{4}$ and $p_{5}$ and its numerical approximations for $\tilde{e}=0 \%$ (a,c,e) and for different noise levels (b,d,f). The relaxation parameter $\omega=10$. 


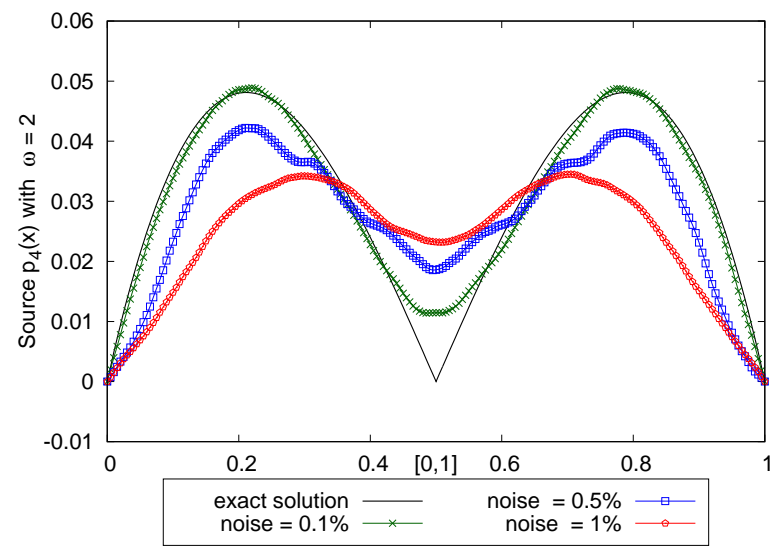

(a)

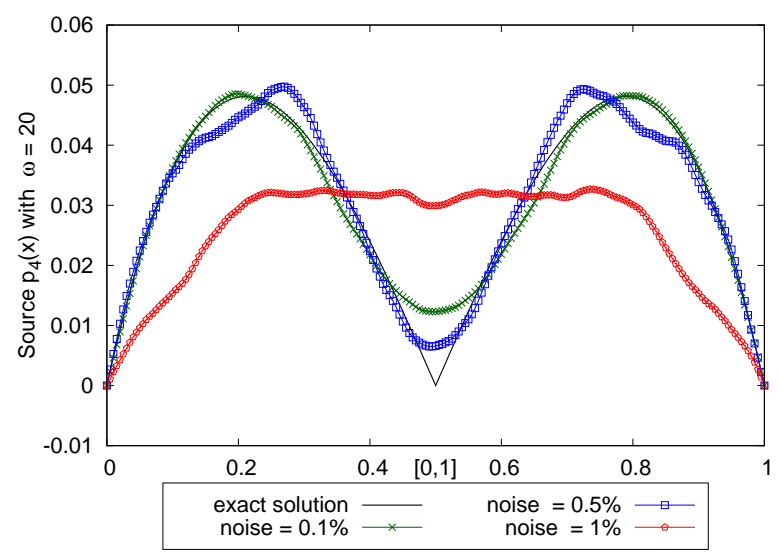

(b)

Figure 4: Example 2: The exact source $p_{4}$ and its numerical approximations for $\omega=2$ (a) and for $\omega=20$ (b).

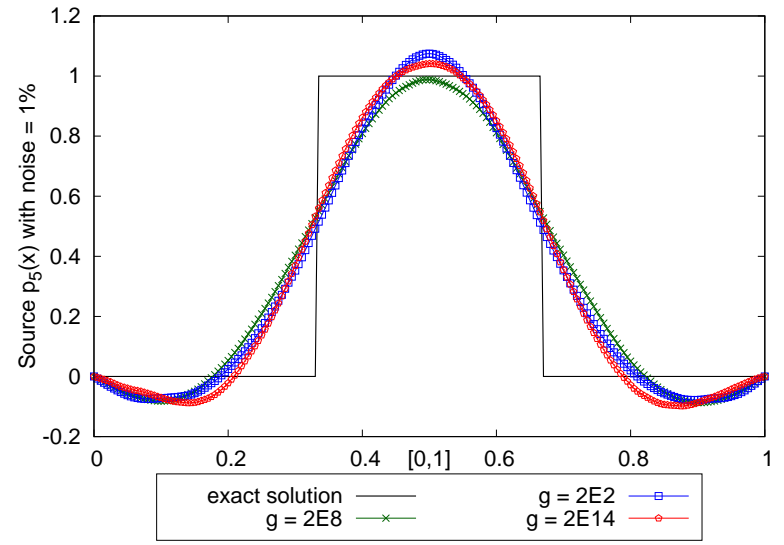

(a)

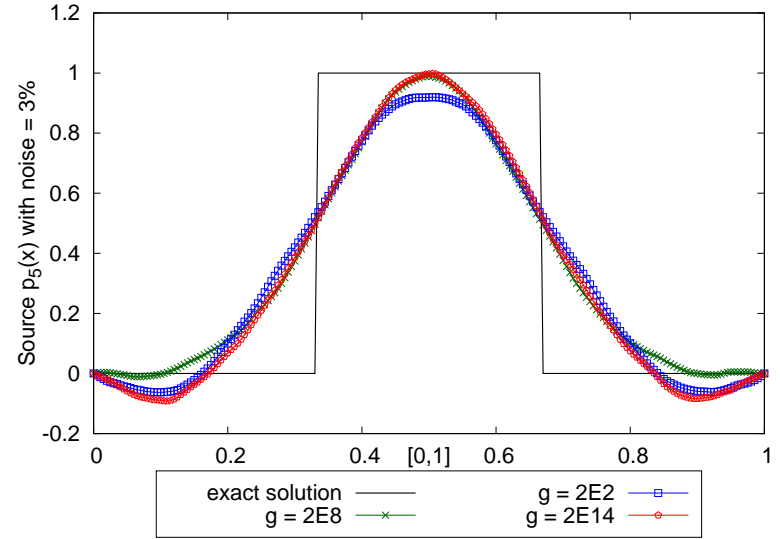

(b)

Figure 5: Example 2: The exact source $p_{5}$ and its numerical approximations for $\tilde{e}=1 \%$ (a) and for $\tilde{e}=3 \%$ (b) for different values of $g$. 


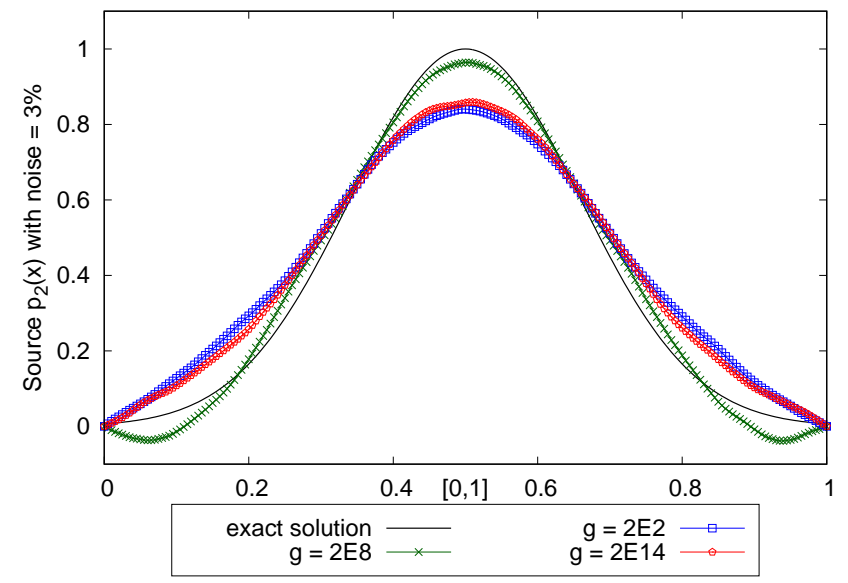

Figure 6: Example 1: The exact source $p_{2}$ and its numerical approximations for $\tilde{e}=3 \%$ for different values of $g$.

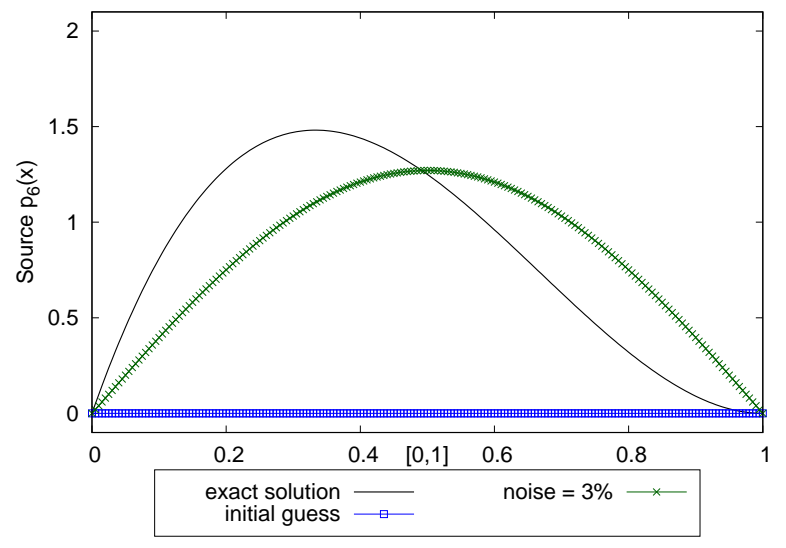

(a)

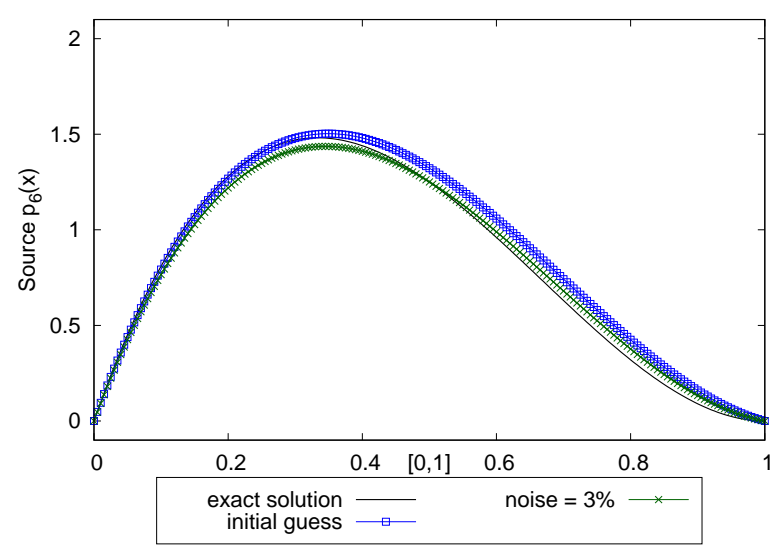

(c)

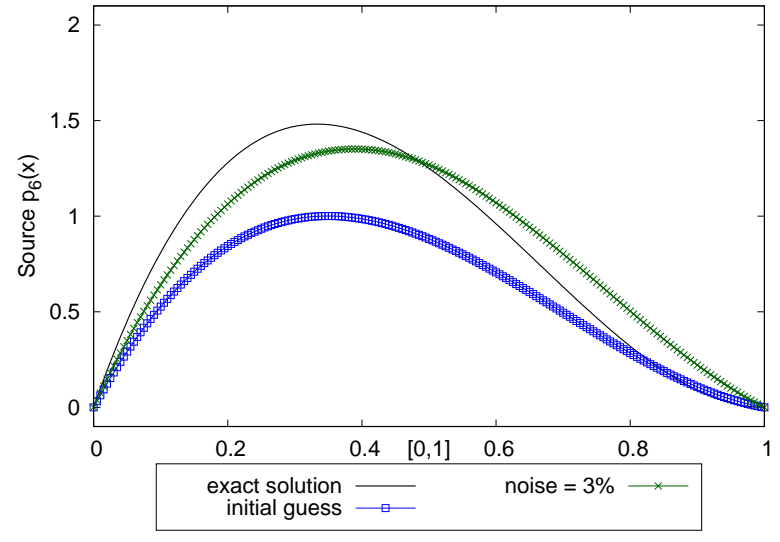

(b)

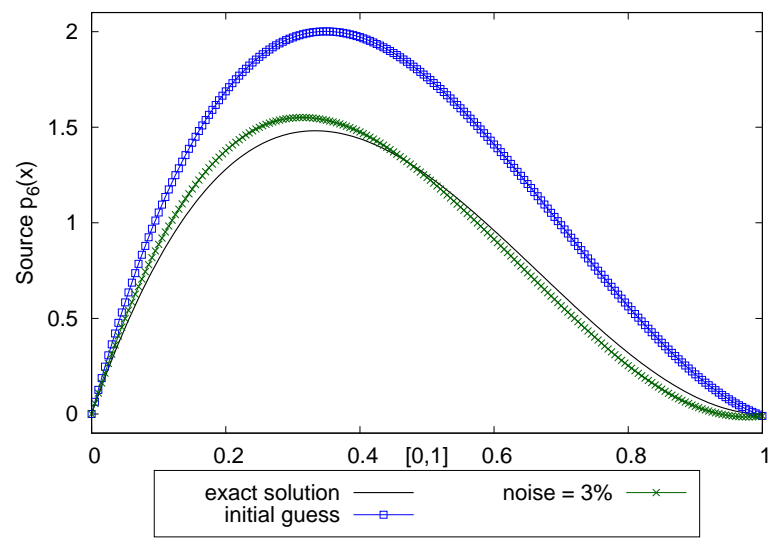

(d)

Figure 7: Example 2: The non-symmetric exact source $p_{6}$ and its numerical approximations (using $\tilde{e}=3 \%$ ) for different initial guesses: 0 (a), $6.44 x-12.27 x^{2}+5.83 x^{3}$ (b), $9.68 x-18.46 x^{2}+8.78 x^{3}$ (c) and $12.88 x-24.54 x^{2}+11.65 x^{3}$ (d). The relaxation parameter $\omega=10$. 


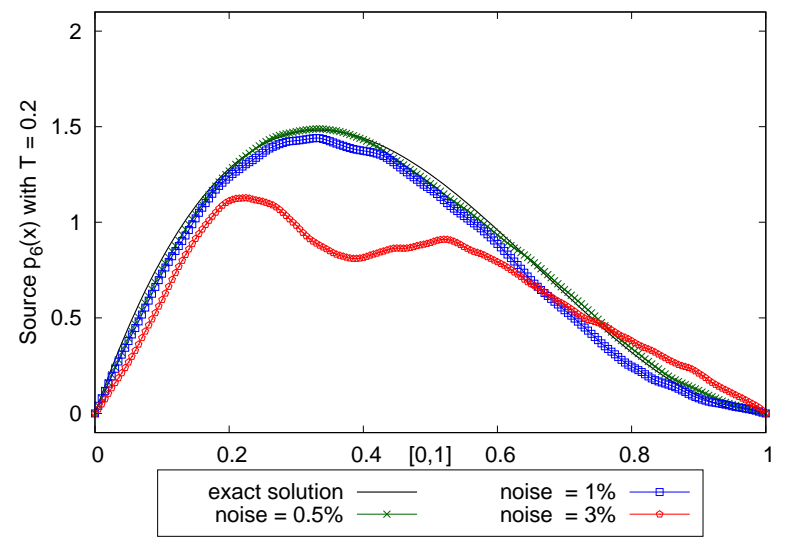

(a)

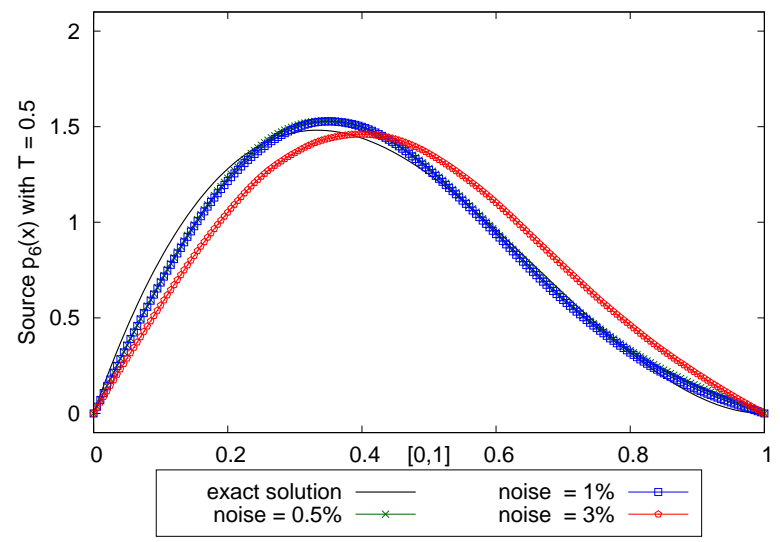

(b)

Figure 8: Example 2: The non-symmetric exact source $p_{6}$ and its numerical approximations for $T=0.2$ (a) and $T=0.5$ (b). The relaxation parameter $\omega=10$.

Table 3: The error $e(\tilde{e}) \approx\left\|\boldsymbol{\xi}_{T}-\boldsymbol{\xi}_{T}^{e}\right\|$, the stopping iteration number $\tilde{k}=k(e(\tilde{e}), 50)$ and the CPU time (mins), obtained for Experiment 3.

\subsection{Experiment 3: $2 D$ problem in a $3 D$ setting}

The 2D linear model an isotropic thermoelastic material in the rectangular domain $\Omega=(0,1) \times(0,1)$, with $K=0$, is given by: Find $(\mathbf{u}, \theta, \mathbf{p})$ such that

$$
\begin{cases}\partial_{t t} \mathbf{u}(\mathbf{x}, t)+g_{2} \partial_{t} \mathbf{u}(\mathbf{x}, t)-\mu_{1} \Delta \mathbf{u}(\mathbf{x}, t)-\left(1-\mu_{1}\right) \nabla(\nabla \cdot \mathbf{u}(\mathbf{x}, t))+\nabla \theta(\mathbf{x}, t)=\mathbf{r}(\mathbf{x}, t)+\mathbf{p}(\mathbf{x}), & (\mathbf{x}, t) \in Q_{T}, \\ \theta_{t}(\mathbf{x}, t)-\Delta \theta(\mathbf{x}, t)+\varepsilon_{2} \nabla \cdot \partial_{t} \mathbf{u}(\mathbf{x}, t)=h(\mathbf{x}, t), & (\mathbf{x}, t) \in Q_{T}, \\ \mathbf{u}(\mathbf{x}, t)=\mathbf{0}, & (\mathbf{x}, t) \in \Sigma_{T}, \\ \theta(\mathbf{x}, t)=0 & (\mathbf{x}, t) \in \Sigma_{T}, \\ \mathbf{u}(\mathbf{x}, 0)=\overline{\mathbf{u}}_{0}(\mathbf{x}), \quad \mathbf{u}_{t}(\mathbf{x}, 0)=\overline{\mathbf{u}}_{1}(\mathbf{x}), & \mathbf{x} \in \Omega, \\ \theta(\mathbf{x}, 0)=\bar{\theta}_{0}(\mathbf{x}), & \mathbf{x} \in \Omega\end{cases}
$$

and the final over-determination condition is satisfied

$$
\mathbf{u}(\mathbf{x}, T)=\boldsymbol{\xi}_{T}^{e}(\mathbf{x}), \quad \mathbf{x} \in \Omega
$$

with

$$
\mu_{1}=\frac{\mu}{\lambda+2 \mu} .
$$


Numerical solution for the first component of $\mathbf{p}$

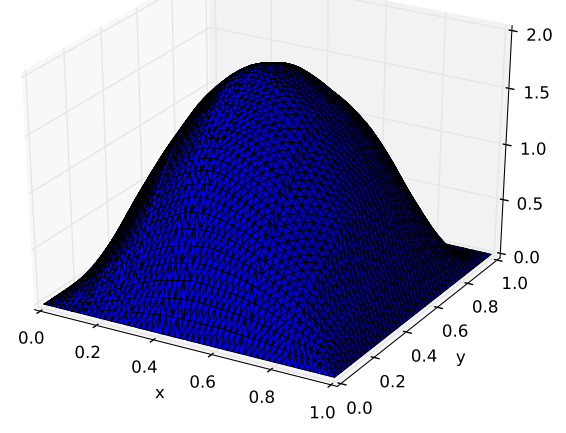

(a)

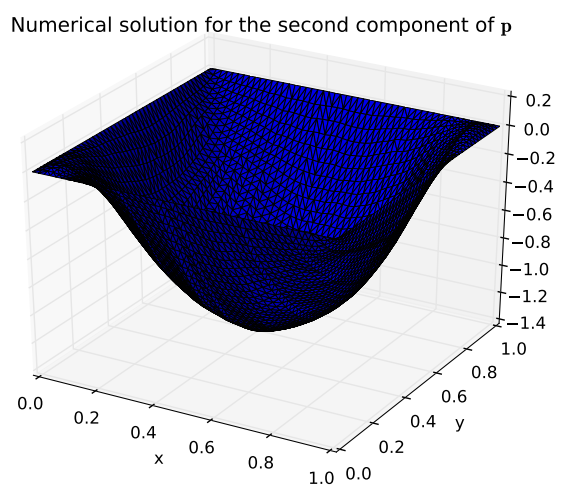

(c)
Pointwise normalized relative errors for the first component of $\mathbf{p}$

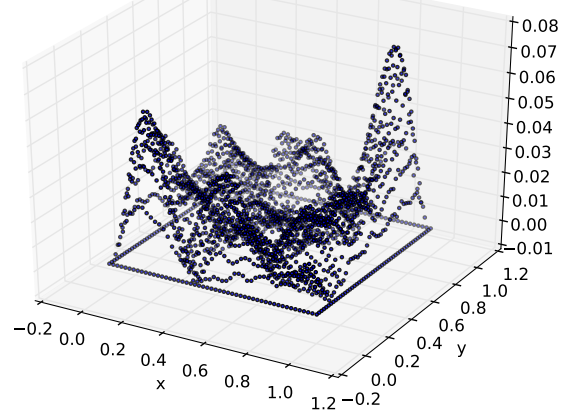

(b)

Pointwise normalized relative errors for the second component of $\mathbf{p}$

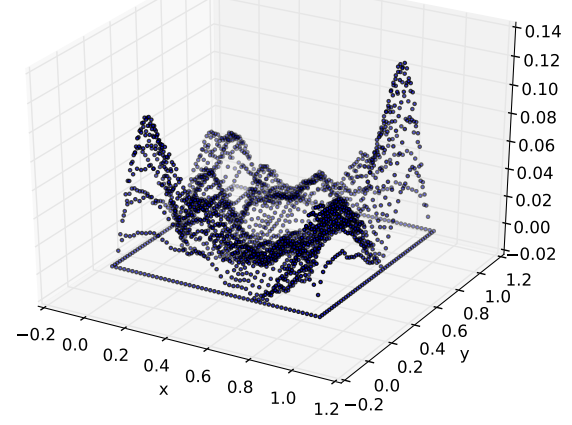

(d)

Figure 9: Example 3: The numerically reconstructed components of the source and their corresponding pointwise normalized relative errors, retrieved using $\tilde{e}=1 \%$. 
Numerical solution for the first component of $p$

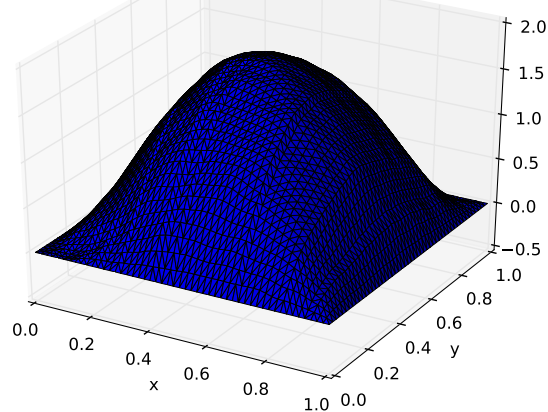

(a)

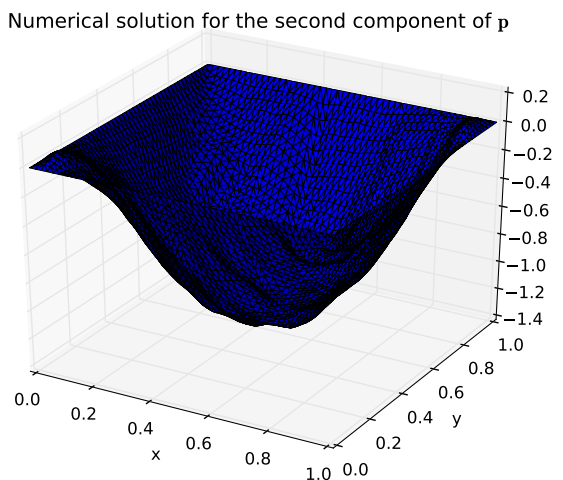

(c)
Pointwise normalized relative errors for the first component of $\mathbf{p}$

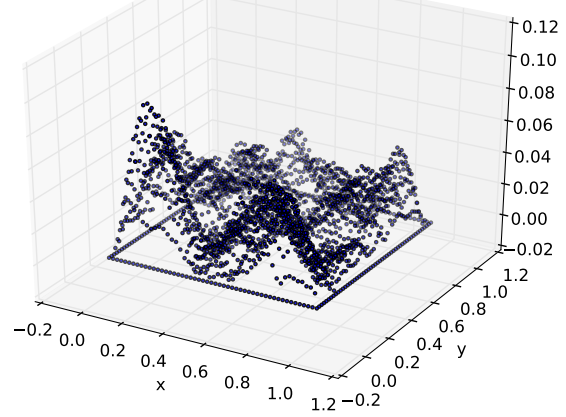

(b)

Pointwise normalized relative errors for the second component of $\mathbf{p}$

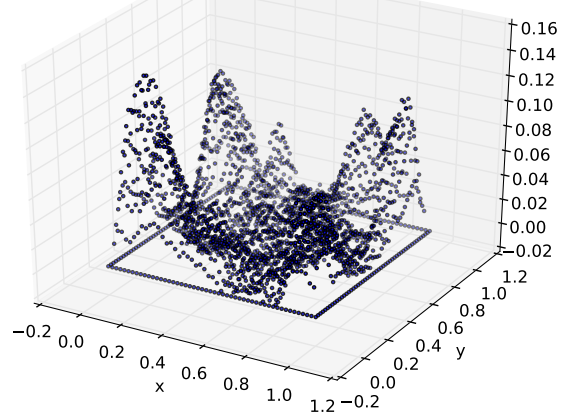

(d)

Figure 10: Example 3: The numerically reconstructed components of the source and their corresponding pointwise normalized relative errors, retrieved using $\tilde{e}=5 \%$. 
The data for the numerical experiment is derived from the following exact solution to problem (37)-(38), which is chosen in accordance to [38, Proposition 1]:

$$
\begin{aligned}
& \mathbf{u}(x, y, t)=(1+t)^{2} \frac{C_{d}}{2}\left[\ln \left((x+5)^{2}+(y+1)^{2}\right)(x+5), \ln \left((x+5)^{2}+(y+1)^{2}\right)(y+1)\right]^{\top}, \\
& \theta(x, y, t)=\left(\frac{1+t}{2}\right) \ln \left((x+5)^{2}+(y+1)^{2}\right), \\
& \mathbf{p}(x, y)=[30 x(x-1) y(y-1),-20 x(x-1) y(y-1)]^{\top},
\end{aligned}
$$

where

$$
C_{d}:=\frac{\alpha_{T}}{2}\left(\frac{1+\nu}{1-\nu}\right) .
$$

The error $e$, the stopping iteration number and the CPU time (in minutes) for this experiment are given in Table 3, for various levels of noise. For each component of $\mathbf{p}$, the numerical results corresponding to $\tilde{e}=1 \%$ and $\tilde{e}=5 \%$ are depicted in Figures 9 and 10 , respectively. Also, the pointwise normalized relative error for each component is shown in these figures. Taking into account the complexity of the problem, we may conclude that good numerical results for the source recovery have been obtained for the $2 \mathrm{D}$ cases investigated.

\section{Conclusion}

In this paper, an inverse source problem associated with a type-III anisotropic thermoelastic system was considered. More precisely, the theoretical and numerical determination of a space-dependent vector source (load) in an anisotropic thermoelastic system of type-III was studied from the knowledge of a supplementary measurement at the final time. The uniqueness of a solution is proved for various assumptions made on the convolution kernel, namely (i) a singular and positive definite convolution kernel; (ii) a singular convolution kernel with the $L_{1}$-norm bounded by a critical constant; and (iii) a bounded convolution kernel, respectively. A convergent and stable iterative algorithm was proposed for the recovery of the unknown vector source in the linear case, whilst a stopping criterion was also given. The numerical experiments carried out herein were implemented using the FEM and validated the convergence and stability of the proposed iterative procedure, as well as the regularizing/stabilizing character of the corresponding stopping criterion. In these experiments, it was showed that the procedure proposed herein is applicable to the reconstruction of symmetric continuous piecewise smooth load fields and symmetric load fields with discontinuities. However, experiments also showed that there is a certain limitation of the method with respect to the recovery of non-symmetric sources wherein only a good approximation is obtained for some specific initial guesses or a smaller final time, which gives a first direction for future research. Other future work will be concerned with the determination of more general (also time-dependent) loadings, the consideration of more general boundary conditions and the implementation of additional experiments.

Acknowledgements. L. Marin acknowledges the financial support received from the Romanian National Authority for Scientific Research (CNCS-UEFISCDI), project number PN-II-ID-PCE-2011-3-0521. The authors would also like to thank Professor Marian Slodička of Ghent University for his encouragement in performing this research work as well as all fruitful discussions carried out. The authors thank the reviewers for their comments and suggestions which helped us improve the quality of the paper. 


\section{References}

[1] W. Nowacki. Thermoelasticity. Pergamon Press, Oxford, 2008.

[2] A. E. Green and P. M. Naghdi. A re-examination of the basic postulates of themomechanics. Proc. Royal Society London A, 432(1885):171-194, 1991.

[3] J. Hadamard. Lectures on Cauchy Problem in Linear Partial Differential Equations. Yale University Press, New Haven, 1923.

[4] B. H. Dennis and G. S. Dulikravich. Simultaneous Determination of Temperatures, Heat Fluxes, Deformations, and Tractions on Inaccessible Boundaries. ASME Journal of Heat Transfer, 121:537$545,1999$.

[5] B. H. Dennis, S. Yoshimura, and G. S. Dulikravich. A Finite Element Formulation for the Determination of Unknown Boundary Conditions for 3-D Steady Thermoelastic Problems. ASME Journal of Heat Transfer, 126:110-118, 2004.

[6] L. Marin and A. Karageorghis. The MFS for the Cauchy problem in two-dimensional steady-state linear thermoelasticity. International Journal of Solids and Structures, 50(20-21):3387-3398, 2013.

[7] A. Karageorghis, D. Lesnic, and L. Marin. The method of fundamental solutions for an inverse boundary value problem in static thermo-elasticity. Computers \& Structures, 135:32-39, 2014.

[8] L. Marin, A. Karageorghis, and D. Lesnic. Regularized MFS solution of inverse boundary value problems in three-dimensional steady-state linear thermoelasticity. International Journal of Solids and Structures, 91:127-142, 2016.

[9] X. Yu. An inverse problem for the quasistatic thermoelastic system on the unit disk. Applicable Analysis, 73(1-2):295-305, 1999.

[10] B. H. Dennis, W. Jin, G. S. Dulikravich, and Jaric. J. Application of the Finite Element Method to Inverse Problems in Solid Mechanics. International Journal of Structural Changes in Solids, 3(2):11$21,2011$.

[11] R. Nedin, S. Nesterov, and A. Vatulyan. On an inverse problem for inhomogeneous thermoelastic rod. International Journal of Solids and Structures, 51(34):767-773, 2014.

[12] M. Bellassoued and M. Yamamoto. Carleman estimates and an inverse heat source problem for the thermoelasticity system. Inverse Problems, 27(1):015006, 2011.

[13] B. Wu and J. Liu. Determination of an unknown source for a thermoelastic system with a memory effect. Inverse Problems, 28(9):095012, 2012.

[14] K. Van Bockstal and M. Slodička. Recovery of a space-dependent vector source in thermoelastic systems. Inverse Problems Sci. Eng., 23(6):956-968, 2015.

[15] B. Wu, J. Yu, and Z. Wang. Uniqueness and stability of an inverse kernel problem for type-III thermoelasticity. J. Math. Anal. and Appl., 402(1):242-254, 2013.

[16] K. Van Bockstal and M. Slodička. Recovery of a time-dependent heat source in one-dimensional thermoelasticity of type-III. Inverse Problems in Science and Engineering, 25(5):749-770, 2017. 
[17] M. Tanaka, A. Guzik, T. Matsumoto, and R. A. Białecki. An inverse estimation of multi-dimensional load distributions in thermoelasticity problems via dual reciprocity BEM. Computational Mechanics, 37:86-95, 2007.

[18] N. Kikuchi and J.T. Oden. Contact Problems in Elasticity: A Study of Variational Inequalities and Finite Element Methods. Studies in Applied Mathematics. Society for Industrial and Applied Mathematics (SIAM, 3600 Market Street, Floor 6, Philadelphia, PA 19104), 1988.

[19] Y. Qin. Nonlinear parabolic-hyperbolic coupled systems and their attractors. Basel: Birkhäuser, 2008.

[20] R. C. MacCamy and J. S. W. Wong. Stability theorems for some functional equations. Transactions of the American Mathematical Society, 164:1-37, 1972.

[21] P. Cannarsa and D. Sforza. Integro-differential equations of hyperbolic type with positive definite kernels. Journal of Differential Equations, 250(12):4289 - 4335, 2011.

[22] J. Kačur. Method of Rothe in evolution equations, volume 80 of Teubner Texte zur Mathematik. Teubner, Leipzig, 1985.

[23] L. C. Evans. Partial differential equations, volume 19 of Graduate Studies in Mathematics. Providence, RI: American Mathematical Society, USA, 1998.

[24] K. Van Bockstal. Numerical techniques for partial differential equations in superconductivity and thermoelasticity. PhD thesis, Ghent University, Ghent, 2015.

[25] T. Roubíček. Nonlinear partial differential equations with applications, volume 153 of ISNM. Birkhäuser Verlag, Basel, 2005.

[26] L. Landweber. An iteration formula for fredholm integral equations of the first kind. American Journal of Mathematics, 73(3):615-624, 1951.

[27] V. M. Fridman. Method of successive approximations for a Fredholm integral equation of the 1st kind. Uspekhi Mat. Nauk, 11(1(67)):233-234, 1956.

[28] T. Johansson and D. Lesnic. Determination of a spacewise dependent heat source. J. Comput. Appl. Math., 209(1):66-80, December 2007.

[29] B. T. Johansson and D. Lesnic. A procedure for determining a spacewise dependent heat source and the initial temperature. Applicable Analysis, 87(3):265-276, 2008.

[30] S. D'haeyer, B. T. Johansson, and M. Slodička. Reconstruction of a spacewise-dependent heat source in a time-dependent heat diffusion process. IMA Journal of Applied Mathematics, 79(1):33-53, 2014.

[31] M. Slodička and V. Melicher. An iterative algorithm for a Cauchy problem in eddy-current modelling. Appl. Math. Comput., 217(1):237-246, 2010.

[32] V. A. Morozov. On the solution of functional equations by the method of regularization. Soviet Math. Dokl., 7:414-417, 1966.

[33] R.B. Ross. Metallic materials specification handbook. Spon, 1980. 
[34] A. Logg and G. N. Wells. DOLFIN: Automated Finite Element Computing. ACM Trans. Math. Software, 37(2):28, 2010.

[35] A. Logg, G.N. Wells, and J. Hake. DOLFIN: a C++/Python Finite Element Library, chapter 10. Springer, Berlin, Heidelberg, 2012.

[36] A. Logg, K-A. Mardal, G. N. Wells, et al. Automated Solution of Differential Equations by the Finite Element Method. Springer, Berlin, Heidelberg, 2012.

[37] M. S. Alnæs, J. Blechta, J. Hake, A. Johansson, B. Kehlet, A. Logg, C. Richardson, J. Ring, M. E. Rognes, and G. N. Wells. The FEniCS project version 1.5. Archive of Numerical Software, 3(100), 2015.

[38] L. Marin, A. Karageorghis, and D. Lesnic. A numerical study of the svdmfs solution of inverse boundary value problems in two-dimensional steady-state linear thermoelasticity. Numerical Methods for Partial Differential Equations, 31(1):168-201, 2015. 